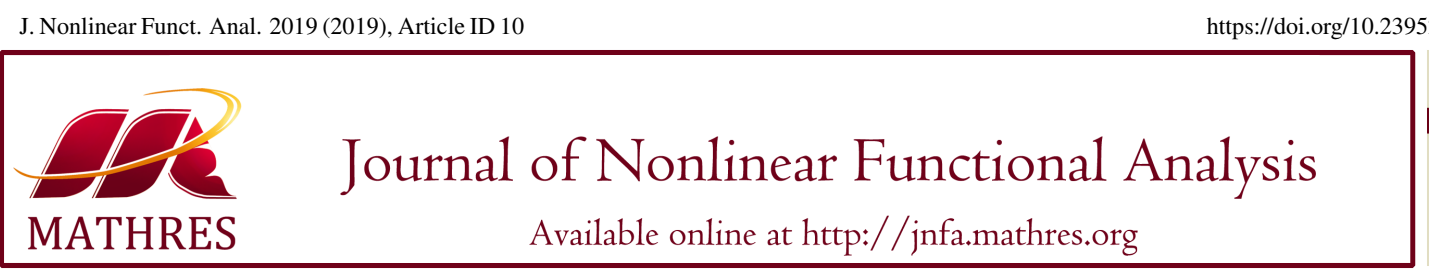

https://doi.org/10.23952/jnfa.2019.10

\title{
GREEN'S FUNCTION APPROACH TO A LARGE TIME BEHAVIOR PROBLEM FOR A COUPLED SYSTEM OF 1-D WAVE AND PLATE EQUATIONS
}

\author{
CHENGQIANG WANG \\ School of Mathematics, Chengdu Normal University, Chengdu 611130, China
}

\begin{abstract}
We study a system composed of a 1-D damped wave equation and a 1-D undamped plate equation, in which the energy of the two parts can be transmitted through the boundary. We show that the spectrum of the infinitesimal generator of the semigroup associated to a system equivalent to the concerned system is contained in the open left half complex plane, and prove by establishing a resolvent estimate on the afore-mentioned infinitesimal generator that the energy of the system under consideration decays at a logarithmic rate. Green's function and Young's inequality are the two main ingredients in proving the afore-mentioned resolvent estimate.
\end{abstract}

Keywords. Wave equation; Plate equation; Spectral property; Logarithmic decay; Green's functions.

2010 Mathematics Subject Classification. 35B35, 35B40, 35P05.

\section{INTRODUCTION}

To suppress of the vibration of elastic bodies, engineers and material scientists usually bond some functional patches to the underlying elastic bodies as active damping "controllers"; see [1]. In this paper, we are concerned with a model of a elastic body whose vibration is suppressed by a functional patch whose energy is inhibited by a linear frictional damping, namely, the transmission system

$$
\left.\begin{array}{ll}
\partial_{t}^{2} u-\partial_{x}^{2} u+a \partial_{t} u=0 & \text { in }(0,1) \times(0,+\infty), \\
\partial_{t}^{2} w+\partial_{x}^{4} w=0 & \text { in }(-1,0) \times(0,+\infty), \\
u(1, t)=\partial_{x}^{2} w(0, t)=w(-1, t)=\partial_{x}^{2} w(-1, t)=0 & \text { for } t \in(0,+\infty), \\
u(0, t)=w(0, t), \partial_{x} u(0, t)=-\partial_{x}^{3} w(0, t) & \text { for } t \in(0,+\infty), \\
u(\cdot, 0)=u^{0}, \partial_{t} u(\cdot, 0)=u^{1} & \text { in }(0,1), \\
w(\cdot, 0)=w^{0}, \partial_{t} w(\cdot, 0)=w^{1} & \text { in }(-1,0),
\end{array}\right\}
$$

where the equation $\partial_{t}^{2} w+\partial_{x}^{4} w=0$ is used to describe the vibration of the elastic body, while the equation $\partial_{t}^{2} u-\partial_{x}^{2} u+a \partial_{t} u=0$ is incorporated to characterize the suppression of the functional patch. In this

E-mail address: cqwung@ foxmail.com.

Received October 10, 2018; Accepted February 22, 2019.

(C)2019 Journal of Nonlinear Functional Analysis 
model, constant $a>0$ reflects the intensity of the frictional damping; the vibration of the elastic body is suppressed by the functional patch via the the "law": $u(0, t)=w(0, t), \partial_{x} u(0, t)=-\partial_{x}^{3} w(0, t)$.

Aside from the above model, transmission systems of partial differential equations can be widely used to describe such diverse physical processes as fluid-structure interactions, electromagnetic coupling, coupled chemical reactions and so on. Therefore, these systems have been extensively investigated in the literature; see $[1,2,3,4]$ and the references therein. Our main goal in this paper is to understand better the longtime behavior of solutions to system (1.1), and our study has been motivated by some newly obtained results in this research field. Ammari and Nicaise [5] proved under a certain geometric condition that the energy of the transmission system of a damped wave equation and a damped plate equation decayed exponentially by a multiplier argument. Zhang and Zhang [6] studied the stabilization of transmission coupled wave and Euler-Bernoulli equations on Riemannian manifolds by introducing nonlinear feedbacks. Hassine [7] proved by the Carleman estimate an energy decay estimate for the transmission system of an undamped Euler-Bernoulli plate and a wave equation with a localized KelvinVoigt damping. Fu and Lü [8] considered a weakly coupled system wave-plate equations, where a localized damping is added either in the wave part or plate part, and proved under some condition on the damping and the coupling terms that sufficiently smooth solutions of the system decayed logarithmically at infinity without any geometric conditions on the effective damping domain. Gong, Yang and Zhao [9] studied the stabilization of a wave/plate transmission system via an Riemannian geometric approach. The controllability of wave/plate transmission systems was also investigated in the literature; see [10] and the references. Inspired by the afore-mentioned results, and in view of the presence of the damping in the wave equation, we are interested in the large-time behavior of solutions to system (1.1).

To study the dynamics of system (1.1), we introduce the auxiliary function $v(x, t)=w(-x, t)$, and plug it into (1.1) to yield a system, which is equivalent to (1.1), that is,

$$
\begin{array}{ll}
\partial_{t}^{2} u-\partial_{x}^{2} u+a \partial_{t} u=0 & \text { in }(0,1) \times(0,+\infty), \\
\partial_{t}^{2} v+\partial_{x}^{4} v=0 & \text { in }(0,1) \times(0,+\infty), \\
u(1, t)=\partial_{x}^{2} v(0, t)=v(1, t)=\partial_{x}^{2} v(1, t)=0 & \text { for } t \in(0,+\infty), \\
u(0, t)=v(0, t), \partial_{x} u(0, t)=\partial_{x}^{3} v(0, t) & \text { for } t \in(0,+\infty), \\
u(\cdot, 0)=u^{0}, \partial_{t} u(\cdot, 0)=u^{1} & \text { in }(0,1), \\
v(\cdot, 0)=v^{0}, \partial_{t} v(\cdot, 0)=v^{1} & \text { in }(0,1) .
\end{array}
$$

Therefore, the study of the longtime behavior of system (1.1) is equivalent to that of system (1.2).

Let us now introduce the space

$$
\begin{gathered}
\mathscr{H}=\left\{(\varphi, \widetilde{\varphi}, \psi, \widetilde{\psi}) \in H^{1}(0,1) \times L^{2}(0,1) \times H^{2}(0,1) \times L^{2}(0,1) ;\right. \\
\varphi(1)=\psi(1)=\varphi(0)-\psi(0)=0\} .
\end{gathered}
$$

Indeed, $\mathscr{H}$, equipped with the sesquilinear form

$$
\mathscr{H}^{2} \ni((\varphi, \widetilde{\varphi}, \psi, \widetilde{\psi}),(f, \widetilde{f}, g, \widetilde{g})) \mapsto \int_{0}^{1}\left(\varphi^{\prime} \overline{f^{\prime}}+\widetilde{\varphi} \overline{\widetilde{f}}+\psi^{\prime \prime} \overline{g^{\prime \prime}}+\widetilde{\psi} \overline{\widetilde{g}}\right) d x \in \mathbb{C}
$$

where $\bar{z}$ denotes the complex conjugate of $z \in \mathbb{C}$, is a Hilbert space. $\mathscr{H}$ is the so-called finite energy space of system (1.2). And, $E(t)$ (resp. $\tilde{E}(t))$ is the natural energy functional associated to system (1.1) 
(resp. (1.2)), where $E(t)$ and $\tilde{E}(t)$ are defined respectively by

$$
\begin{aligned}
& E(t)=\frac{1}{2} \int_{0}^{1}\left(\left|\partial_{t} u(x, t)\right|^{2}+\left|\partial_{x} u(x, t)\right|^{2}\right) d x+\frac{1}{2} \int_{-1}^{0}\left(\left|\partial_{t} w(x, t)\right|^{2}+\left|\partial_{x}^{2} w(x, t)\right|^{2}\right) d x \quad \text { and } \\
& \tilde{E}(t)=\frac{1}{2} \int_{0}^{1}\left(\left|\partial_{t} u(x, t)\right|^{2}+\left|\partial_{x} u(x, t)\right|^{2}\right) d x+\frac{1}{2} \int_{0}^{1}\left(\left|\partial_{t} v(x, t)\right|^{2}+\left|\partial_{x}^{2} v(x, t)\right|^{2}\right) d x
\end{aligned}
$$

Let us define an unbounded linear operator $A$ in space $\mathscr{H}$ by

$$
\left.\begin{array}{l}
A(\varphi, \widetilde{\varphi}, \psi, \widetilde{\psi})=\left(\widetilde{\varphi}, \varphi^{\prime \prime}-a \widetilde{\varphi}, \widetilde{\psi},-\psi^{(4)}\right), \quad \forall(\varphi, \widetilde{\varphi}, \psi, \widetilde{\psi}) \in \mathscr{D}(A), \\
\mathscr{D}(A)=\left\{(\varphi, \widetilde{\varphi}, \psi, \widetilde{\psi}) \in \mathscr{H} \cap\left(H^{2}(0,1) \times H^{1}(0,1) \times H^{4}(0,1) \times H^{2}(0,1)\right) ;\right. \\
\widetilde{\varphi}(1)=\widetilde{\psi}(1)=\widetilde{\varphi}(0)-\widetilde{\psi}(0)=\psi^{\prime \prime}(0) \\
\left.=\psi^{\prime \prime}(1)=\varphi^{\prime}(0)-\psi^{\prime \prime \prime}(0)=0\right\} .
\end{array}\right\}
$$

The following is one of our main results.

\section{Theorem 1.1.}

- A is the infinitesimal generator of a strongly continuous semigroup of contractions on $\mathscr{H}$.

- $\sigma(A)$ is contained in the open left half complex plane $\mathbb{C}^{-}$and consists merely of eigenvalues $\left\{\lambda_{1 k}, \lambda_{2 \ell}\right\}$ of $A$. And moreover, the eigenvalues are distributed as follows:

$$
\left.\begin{array}{l}
\lambda_{1 k}=\frac{-a \pm \mathrm{i} \sqrt{4 k^{2} \pi^{2}-a^{2}}}{2}+\mathscr{O}\left(k^{-\frac{1}{2}}\right) \quad \text { as } k \rightarrow+\infty \text { with } k \in \mathbb{N}, \\
\lambda_{2 \ell}= \pm \pi^{2}\left(\ell+\frac{1}{4}\right)^{2} \mathrm{i}+\mathscr{O}\left(|\ell|^{-\frac{1}{2}}\right) \quad \text { as }|\ell| \rightarrow+\infty \text { with } \ell \in \mathbb{Z} .
\end{array}\right\}
$$

The fact that $\sigma(A)$ is contained in the open left half complex plane implies that energy $\tilde{E}(t)$ (or equivalently $E(t))$ decreases to 0 as $t \rightarrow+\infty$; see $[11,12,13]$ and the references therein. Motivated by this, we study operator $A$ and obtain the following logarithmic decaying estimate on $E(t)$.

Theorem 1.2. There exists a $C>0$ such that, for every quadruple $\left(u^{0}, u^{1}, w^{0}, w^{1}\right) \in \mathscr{D}(A)$,

$$
E(t) \leqslant \frac{C}{\ln ^{2}(2+t)}\left(\left\|u^{0}\right\|_{H^{2}(0,1)}^{2}+\left\|u^{1}\right\|_{H^{1}(0,1)}^{2}+\left\|w^{0}\right\|_{H^{4}(-1,0)}^{2}+\left\|w^{1}\right\|_{H^{2}(-1,0)}^{2}\right), \quad \forall t \in[0,+\infty) .
$$

Note that $E(t)$ and $\tilde{E}(t)$ share the same decaying rate. To obtain a decaying estimate on $\tilde{E}(t)$, we analyze in detail the resolvent $\left(\lambda \operatorname{id}_{\mathscr{H}}-A\right)^{-1}$ and obtain an useful estimate on it along the imaginary axis. Green's function and Young's inequality are key ingredients in obtaining the afore-claimed resolvent estimate.

The rest of the paper is planned as follows. In Section 2, we prove Theorem 1.1. In Section 3, we prove Theorem 1.2. In last section, Section 4, a concluding remark is provided.

\section{Proof of Theorem 1.1}

The principal goal of this section is to analyze in detail the spectral structure of $A$. First, we prove that IBVP (1.2) is well-posed in $\mathscr{H}$.

Proposition 2.1. $A$ is the infinitesimal generator of a strongly continuous semigroup of contractions $\left\{e^{t A}\right\}_{t \in[0,+\infty)}$ on $\mathscr{H}$. 
Proof. Define an unbounded linear operator $A^{*}$ in $\mathscr{H}$ by

$$
\left.\begin{array}{l}
A^{*}(\varphi, \widetilde{\varphi}, \psi, \widetilde{\psi})=\left(-\widetilde{\varphi},-\varphi^{\prime \prime}-a \widetilde{\varphi},-\widetilde{\psi}, \psi^{(4)}\right), \quad \forall(\varphi, \widetilde{\varphi}, \psi, \widetilde{\psi}) \in \mathscr{D}\left(A^{*}\right), \\
\mathscr{D}\left(A^{*}\right)=\mathscr{D}(A) .
\end{array}\right\}
$$

Indeed, $A^{*}$ is the adjoint of $A$ in $\mathscr{H}$. By using integration-by-parts, we have

$$
\begin{aligned}
& 2 \Re \mathfrak{e}\left\langle A^{*}(\varphi, \widetilde{\varphi}, \psi, \widetilde{\psi}),(\varphi, \widetilde{\varphi}, \psi, \widetilde{\psi})\right\rangle_{\mathscr{H}} \\
= & \int_{0}^{1}\left[-\widetilde{\varphi}^{\prime}(x) \overline{\varphi^{\prime}(x)}-\varphi^{\prime \prime}(x) \overline{\widetilde{\varphi}(x)}-a|\widetilde{\varphi}(x)|^{2}-\widetilde{\psi}^{\prime \prime}(x) \overline{\psi^{\prime \prime}(x)}+\psi^{(4)}(x) \overline{\widetilde{\psi}(x)}\right] d x \\
& +\int_{0}^{1}\left[-\overline{\widetilde{\varphi}^{\prime}(x)} \varphi^{\prime}(x)-\overline{\varphi^{\prime \prime}(x)} \widetilde{\varphi}(x)-a|\widetilde{\varphi}(x)|^{2}-\overline{\widetilde{\psi}^{\prime \prime}(x)} \psi^{\prime \prime}(x)+\overline{\psi^{(4)}(x)} \widetilde{\psi}(x)\right] d x \\
= & {\left[\psi^{\prime \prime \prime}(x) \overline{\widetilde{\psi}(x)}-\psi^{\prime \prime}(x) \overline{\widetilde{\psi}^{\prime}(x)}+\overline{\psi^{\prime \prime \prime}(x)} \widetilde{\psi}(x)-\overline{\psi^{\prime \prime}(x)} \widetilde{\psi}^{\prime}(x)-\widetilde{\varphi}(x) \overline{\varphi^{\prime}(x)}-\overline{\widetilde{\varphi}(x)} \varphi^{\prime}(x)\right]_{0}^{1} } \\
& -2 a\|\widetilde{\varphi}\|_{L^{2}(0,1)} \\
= & -2 a\|\widetilde{\varphi}\|_{L^{2}(0,1)}, \quad \forall(\varphi, \widetilde{\varphi}, \psi, \widetilde{\psi}) \in \mathscr{D}\left(A^{*}\right),
\end{aligned}
$$

which implies

$$
\mathfrak{R e}\left\langle A^{*}(\varphi, \widetilde{\varphi}, \psi, \widetilde{\psi}),(\varphi, \widetilde{\varphi}, \psi, \widetilde{\psi})\right\rangle_{\mathscr{H}}=-a\|\widetilde{\varphi}\|_{L^{2}(0,1)}^{2}, \quad \forall(\varphi, \widetilde{\varphi}, \psi, \widetilde{\psi}) \in \mathscr{D}\left(A^{*}\right)
$$

Similarly, we can show

$$
\mathfrak{R e}\langle A(\varphi, \widetilde{\varphi}, \psi, \widetilde{\psi}),(\varphi, \widetilde{\varphi}, \psi, \widetilde{\psi})\rangle_{\mathscr{H}}=-a\|\widetilde{\varphi}\|_{L^{2}(0,1)}^{2}, \quad \forall(\varphi, \widetilde{\varphi}, \psi, \widetilde{\psi}) \in \mathscr{D}(A)
$$

In addition, $A$ is densely defined and closed in $\mathscr{H}$. By [14, Corollary 4.4, p. 15], $A$ is the infinitesimal generator of a strongly continuous semigroup of contractions $\left\{e^{t A}\right\}_{t \in[0,+\infty)}$ on $\mathscr{H}$. This completes the proof.

Remark 2.1. In light of (2.1), $A$ is dissipative and therefore $\lambda \notin \sigma(A)$ whenever $\mathfrak{R e} \lambda>0$. In particular, this implies that the resolvent set of $A$ is nonempty.

Remark 2.2. In view of the embedding $\mathscr{D}(A) \subset \mathscr{H}$ by the Rellich-Kondrachov theorem, $A$ has compact resolvents. Therefore, $\sigma(A)$ consists merely of a sequence of eigenvalues.

Next, we prove that $\sigma(A)$ is contained in the open left half complex plane.

Proposition 2.2. $\sigma(A) \subset \mathbb{C}^{-}$, where $\mathbb{C}^{-}=\{\lambda \in \mathbb{C} ; \mathfrak{R e} \lambda<0\}$.

Proof. By Remark 2.1, it suffices to show that $\mathrm{i} \cap \cap \sigma(A)=\emptyset$. Indeed, $0 \notin \sigma(A)$. Otherwise, there exists, by Remark 2.2, a nonzero quadruple $(\varphi, \widetilde{\varphi}, \psi, \widetilde{\psi})$ such that $A(\varphi, \widetilde{\varphi}, \psi, \widetilde{\psi})=\left(\widetilde{\varphi}, \varphi^{\prime \prime}-a \widetilde{\varphi}, \widetilde{\psi},-\psi^{(4)}\right)=$ $0(\varphi, \widetilde{\varphi}, \psi, \widetilde{\psi})$. We have $\widetilde{\varphi}=0, \widetilde{\psi}=0, \varphi^{\prime \prime}=0$ and $\psi^{(4)}=0$. Therefore, $\varphi$ and $\psi$ assume the forms $\varphi(x)=c_{11} x+c_{12}$ and $\psi(x)=c_{21} x^{3}+c_{22} x^{2}+c_{23} x+c_{24}$, respectively. Recalling the boundary conditions 
to which $\varphi$ and $\psi$ are subject, we have

$$
\left.\begin{array}{rl}
\varphi(1)=0 & \Longrightarrow c_{11}+c_{12}=0,, \\
\psi(1)=0 & \Longrightarrow c_{21}+c_{22}+c_{23}+c_{24}=0, \\
\varphi(0)-\psi(0)=0 & \Longrightarrow c_{12}-c_{24}=0, \\
\psi^{\prime \prime}(0)=0 & \Longrightarrow 2 c_{22}=0, \\
\psi^{\prime \prime}(1)=0 & \Longrightarrow 6 c_{21}+2 c_{22}=0, \\
\varphi^{\prime}(0)-\psi^{\prime \prime \prime}(0)=0 & \Longrightarrow c_{11}-6 c_{21}=0 .
\end{array}\right\}
$$

It is ready to check that

$$
\operatorname{det}\left(\begin{array}{cccccc}
1 & 1 & 0 & 0 & 0 & 0 \\
0 & 0 & 1 & 1 & 1 & 1 \\
0 & 1 & 0 & 0 & 0 & -1 \\
0 & 0 & 0 & 2 & 0 & 0 \\
0 & 0 & 6 & 2 & 0 & 0 \\
1 & 0 & -6 & 0 & 0 & 0
\end{array}\right)=-12 \neq 0
$$

Then $c_{11}=c_{12}=c_{21}=c_{22}=c_{23}=c_{24}=0$, that is, $\varphi=0$ and $\psi=0$. This, together with the observation that $\widetilde{\varphi}=0$ and $\widetilde{\psi}=0$, contradicts the the fact that quadruple $(\varphi, \widetilde{\varphi}, \psi, \widetilde{\psi})$ is nonzero. That is, $0 \in \rho(A)$.

It remains to show that $\mathrm{i} \mu \notin \sigma(A)$ for every $\mu \in \mathbb{R} \backslash\{0\}$. Let $(\varphi, \widetilde{\varphi}, \psi, \widetilde{\psi}) \in \mathscr{D}(A)$ be such that $A(\varphi, \widetilde{\varphi}, \psi, \widetilde{\psi})=\mathrm{i} \mu(\varphi, \widetilde{\varphi}, \psi, \widetilde{\psi})$ for some $\mu \in \mathbb{R} \backslash\{0\}$. We have

$$
\langle A(\varphi, \widetilde{\varphi}, \psi, \widetilde{\psi}),(\varphi, \widetilde{\varphi}, \psi, \widetilde{\psi})\rangle_{\mathscr{H}}=\mathrm{i} \mu\|(\varphi, \widetilde{\varphi}, \psi, \widetilde{\psi})\|_{\mathscr{H}}^{2}
$$

which implies $\mathfrak{R e}\langle A(\varphi, \widetilde{\varphi}, \psi, \widetilde{\psi}),(\varphi, \widetilde{\varphi}, \psi, \widetilde{\psi})\rangle_{\mathscr{H}}=0$. This, together with (2.1), implies

$$
\mathfrak{R e}\langle A(\varphi, \widetilde{\varphi}, \psi, \widetilde{\psi}),(\varphi, \widetilde{\varphi}, \psi, \widetilde{\psi})\rangle_{\mathscr{H}}=-a\|\widetilde{\varphi}\|_{L^{2}(0,1)}^{2}
$$

We therefore have $\widetilde{\varphi}(x)=0, x \in[0,1]$. Thus, equation $A(\varphi, \widetilde{\varphi}, \psi, \widetilde{\psi})=\mathrm{i} \mu(\varphi, \widetilde{\varphi}, \psi, \widetilde{\psi})$ is reduced to the boundary value problem

$$
\left.\begin{array}{ll}
\psi^{(4)}=\mu^{2} \psi & \text { in }(0,1), \\
\psi(0)=\psi^{\prime \prime}(0)=\psi^{\prime \prime}(1)=\psi^{\prime \prime \prime}(0)=0 . &
\end{array}\right\}
$$

The solution to BVP (2.3) can be written as

$$
\psi(x)=\hat{c}_{1} \cosh (x \sqrt{|\mu|})+\hat{c}_{2} \sinh (x \sqrt{|\mu|})+\hat{c}_{3} \cos (x \sqrt{|\mu|})+\hat{c}_{4} \sin (x \sqrt{|\mu|})
$$

where $\hat{c}_{1}, \hat{c}_{2}, \hat{c}_{3}$ and $\hat{c}_{4}$ are chosen such that

$$
\begin{aligned}
& \hat{c}_{1}+\hat{c}_{3}=0, \\
& \hat{c}_{1}-\hat{c}_{3}=0, \\
& \hat{c}_{1} \cosh (\sqrt{|\mu|})+\hat{c}_{2} \sinh (\sqrt{|\mu|})-\hat{c}_{3} \cos (\sqrt{|\mu|})-\hat{c}_{4} \sin (\sqrt{|\mu|})=0, \\
& \hat{c}_{2}-\hat{c}_{4}=0 .
\end{aligned}
$$

We deduce from the first two equations that $\hat{c}_{1}=\hat{c}_{3}=0$. Substitute this into the third equation, use the fact that $\sinh (\sqrt{|\mu|})>\sqrt{|\mu|}>\sin (\sqrt{|\mu|})$ for $\mu \in \mathbb{R} \backslash\{0\}$, and conduct some routine calculations, to obtain $\hat{c}_{2}=\hat{c}_{4}=0$. Therefore, $\psi(x)=0, x \in[0,1]$. To sum, $(\varphi, \widetilde{\varphi}, \psi, \widetilde{\psi})=0$. This means that $\mathrm{i} \mu \notin \sigma(A)$ for every $\mu \in \mathbb{R} \backslash\{0\}$. The proof is complete. 
Proposition 2.3. Let $\lambda \in \mathbb{R} \backslash\{0\}$. Then $\lambda \in \sigma(A)$ if and only if $\lambda$ satisfies the equation $\Sigma(\lambda)=0$, where

$$
\begin{aligned}
\Sigma(\lambda)= & \lambda \sqrt{\mathrm{i} \lambda} \sinh (\sqrt{\lambda(\lambda+a)})[\cosh (\sqrt{\mathrm{i} \lambda}) \sinh (\mathrm{i} \sqrt{\mathrm{i} \lambda})-\mathrm{i} \cosh (\mathrm{i} \sqrt{\mathrm{i} \lambda}) \sinh (\sqrt{\mathrm{i} \lambda})] \\
& +2 \mathrm{i} \sqrt{\lambda(\lambda+a)} \cosh (\sqrt{\lambda(\lambda+a)}) \sinh (\mathrm{i} \sqrt{\mathrm{i} \lambda}) \sinh (\sqrt{\mathrm{i} \lambda}) .
\end{aligned}
$$

Proof. We should note that $\lambda \in \sigma(A)$ if and only if the following boundary value problem admits a nonzero solution:

$$
\begin{array}{ll}
\lambda^{2} \varphi-\varphi^{\prime \prime}+a \lambda \varphi=0 & \text { in }(0,1), \\
\lambda^{2} \psi+\psi^{(4)}=0 & \text { in }(0,1), \\
\varphi(1)=\psi^{\prime \prime}(0)=\psi(1)=\psi^{\prime \prime}(1)=0, & \\
\varphi(0)=\psi(0), \quad \varphi^{\prime}(0)=\psi^{\prime \prime \prime}(0) . &
\end{array}
$$

By the classical theory of ODEs, $(\varphi, \psi)$ can be written as

$$
\begin{aligned}
\varphi(x)= & c_{1} \cosh ((1-x) \sqrt{\lambda(\lambda+a)})+c_{2} \sinh ((1-x) \sqrt{\lambda(\lambda+a)}), \text { and } \\
\psi(x)= & c_{3} \cosh ((1-x) \sqrt{\mathrm{i} \lambda})+c_{4} \sinh ((1-x) \sqrt{\mathrm{i} \lambda}) \\
& +c_{5} \cosh ((1-x) \mathrm{i} \sqrt{\mathrm{i} \lambda})+c_{6} \sinh ((1-x) \mathrm{i} \sqrt{\mathrm{i} \lambda}) .
\end{aligned}
$$

The coefficients $c_{1}, c_{2}, c_{3}, c_{4}, c_{5}, c_{6}$ are determined by the boundary conditions on $\varphi$ and $\psi$. More precisely, we have

$$
\begin{aligned}
& \varphi(1)=0 \Longrightarrow c_{1}=0 \\
& \psi(1)=0 \Longrightarrow c_{3}+c_{5}=0 \\
& \psi^{\prime \prime}(1)=0 \Longrightarrow c_{3} \mathrm{i} \lambda-c_{5} \mathrm{i} \lambda=0 \\
& \psi^{\prime \prime}(0)=0 \Longrightarrow c_{4} \mathrm{i} \lambda \sinh (\sqrt{\mathrm{i} \lambda})-c_{6} \mathrm{i} \lambda \sinh (\mathrm{i} \sqrt{\mathrm{i} \lambda})=0, \\
& \varphi(0)-\psi(0)=0 \Longrightarrow c_{2} \sinh (\sqrt{\lambda(\lambda+a)})-c_{4} \sinh (\sqrt{\mathrm{i} \lambda})-c_{6} \sinh (\mathrm{i} \sqrt{\mathrm{i} \lambda})=0 \\
& \varphi^{\prime}(0)-\psi^{\prime \prime \prime}(0)=0 \Longrightarrow-c_{2} \sqrt{\lambda(\lambda+a)} \cosh (\sqrt{\lambda(\lambda+a)})+c_{4} \mathrm{i} \lambda \sqrt{\mathrm{i} \lambda} \cosh (\sqrt{\mathrm{i} \lambda}) \\
&+c_{6} \lambda \sqrt{\mathrm{i} \lambda} \cosh (\mathrm{i} \sqrt{\mathrm{i} \lambda})=0 .
\end{aligned}
$$

By the related classical theory from Linear Algebra, Equation (2.5) has a nontrivial solution if and only if

$$
\begin{aligned}
& \operatorname{det}\left(\begin{array}{cccccc}
1 & 0 & 0 & 0 & 0 & 0 \\
0 & 0 & 1 & 0 & 1 & 0 \\
0 & 0 & \mathrm{i} \lambda & 0 & -\mathrm{i} \lambda & 0 \\
0 & 0 & 0 & \mathrm{i} \lambda \sinh (\sqrt{\mathrm{i} \lambda}) & 0 & -\mathrm{i} \lambda \sinh (\mathrm{i} \sqrt{\mathrm{i} \lambda}) \\
0 & \sinh (\sqrt{\lambda(\lambda+a)}) & 0 & -\sinh (\sqrt{\mathrm{i} \lambda}) & 0 & -\sinh (\mathrm{i} \sqrt{\mathrm{i} \lambda}) \\
0-\sqrt{\lambda(\lambda+a)} \cosh (\sqrt{\lambda(\lambda+a)}) & 0 & \mathrm{i} \lambda \sqrt{\mathrm{i} \lambda} \cosh (\sqrt{\mathrm{i} \lambda}) & 0 & \lambda \sqrt{\mathrm{i} \lambda} \cosh (\mathrm{i} \sqrt{\mathrm{i} \lambda})
\end{array}\right) \\
= & 2 \mathrm{i} \lambda^{2} \Sigma(\lambda)=0 .
\end{aligned}
$$

Taking into the fact that $\lambda \neq 0$, we obtain the desired conclusion immediately. 
Proposition 2.4. $\sigma(A)$ is contained in the open left half complex plane $\mathbb{C}^{-}$and consists merely of eigenvalues $\left\{\lambda_{1 k}, \lambda_{2 \ell}\right\}$ of $A$. Moreover, the eigenvalues are distributed as follows:

$$
\left.\begin{array}{l}
\lambda_{1 k}=\frac{-a \pm \mathrm{i} \sqrt{4 k^{2} \pi^{2}-a^{2}}}{2}+\mathscr{O}\left(k^{-\frac{1}{2}}\right) \quad \text { as } k \rightarrow+\infty \text { with } k \in \mathbb{N}, \\
\lambda_{2 \ell}= \pm \pi^{2}\left(\ell+\frac{1}{4}\right)^{2} \mathrm{i}+\mathscr{O}\left(|\ell|^{-\frac{1}{2}}\right) \quad \text { as }|\ell| \rightarrow+\infty \text { with } \ell \in \mathbb{Z} .
\end{array}\right\}
$$

Proof. Recall that $\sigma(A) \subset \mathbb{C}^{-}$. We consider the situation $\lambda \in \mathbb{D}_{1} \cup \mathbb{D}_{2}$, where $\mathbb{D}_{1}=\left\{\lambda \in \mathbb{C}^{-} ; \arg \lambda \in\right.$ $\left.\left[\frac{3 \pi}{4}, \pi\right]\right\}$ and $\mathbb{D}_{2}=\left\{\lambda \in \mathbb{C}^{-} ; \arg \lambda \in\left[\frac{\pi}{2}, \frac{3 \pi}{4}\right]\right\}$. For the sake of simplification, we would use $\lambda=|\lambda| e^{\mathrm{i} \theta}$ for complex numbers. We next study the eigenvalues of $A$ in $\mathbb{D}_{1} \cup \mathbb{D}_{2}$.

Case $1\left(\lambda \in \mathbb{D}_{1}\right)$. By applying Taylor's expansion, we have

$$
e^{\sqrt{\lambda(\lambda+a)}}=e^{\sqrt{\lambda(\lambda+a)}}=e^{-\lambda \sqrt{1+\frac{a}{\lambda}}}=e^{-\lambda\left[1+\frac{a}{2 \lambda}+\mathscr{O}\left(|\lambda|^{-2}\right)\right]}=e^{-\lambda-\frac{a}{2}+\mathscr{O}\left(|\lambda|^{-1}\right)}
$$

for $\lambda$ with sufficiently large modules. In addition, we also have

$$
\begin{aligned}
e^{\sqrt{\mathrm{i} \lambda}} & =\exp \left(\sqrt{|\lambda|} e^{\mathrm{i}\left(\frac{\theta}{2}-\frac{3 \pi}{4}\right)}\right)=\exp \left(\sqrt{|\lambda|} \cos \left(\frac{\theta}{2}-\frac{3 \pi}{4}\right)\right) \exp \left(\mathrm{i} \sqrt{|\lambda|} \sin \left(\frac{\theta}{2}-\frac{3 \pi}{4}\right)\right), \text { and } \\
e^{\mathrm{i} \sqrt{\mathrm{i} \lambda}} & =\exp \left(\sqrt{|\lambda|} \cos \left(\frac{\theta}{2}-\frac{\pi}{4}\right)\right) \exp \left(\mathrm{i} \sqrt{|\lambda|} \sin \left(\frac{\theta}{2}-\frac{\pi}{4}\right)\right) .
\end{aligned}
$$

Therefore,

$$
\lim _{|\lambda| \rightarrow+\infty}\left|e^{\sqrt{\lambda(\lambda+a)}}\right|=+\infty, \quad \quad \lim _{|\lambda| \rightarrow+\infty}\left|e^{\sqrt{\mathrm{i} \lambda}}\right|=+\infty, \text { and } \quad \lim _{|\lambda| \rightarrow+\infty}\left|e^{\mathrm{i} \sqrt{\mathrm{i} \lambda}}\right|=+\infty .
$$

By analyzing the definition (2.4) of $\Sigma(\lambda)$, we have

$$
\lim _{\lambda \in \mathbb{D}_{1},|\lambda| \rightarrow+\infty} \frac{\Sigma(\lambda)}{\lambda \sqrt{\mathrm{i} \lambda} e^{\sqrt{\lambda(\lambda+a)}+\sqrt{\mathrm{i} \lambda}+\mathrm{i} \sqrt{\mathrm{i} \lambda}}}=\frac{1-\mathrm{i}}{8} \neq 0 .
$$

This, together with (2.9), implies that $\lambda \in \mathbb{D}_{1}$ cannot be a solution to equation $\Sigma(\lambda)=0$, or equivalently, $\lambda$ cannot be an eigenvalue of $A$, whenever its module $|\lambda|$ is sufficiently large.

Case $2\left(\lambda \in \mathbb{D}_{2}\right)$. We deduce from (2.6), (2.7), and (2.8) that

$$
\left|e^{-\sqrt{\lambda(\lambda+a)}}\right|=\left|e^{\lambda+\frac{a}{2}+\mathscr{O}\left(|\lambda|^{-1}\right)}\right| \leqslant C_{1},
$$

for some $C_{1}>0$,

$$
\begin{aligned}
& \left|e^{-\sqrt{\mathrm{i} \lambda} \mid}=\right| \exp \left(-\sqrt{|\lambda|} \cos \left(\frac{\theta}{2}-\frac{3 \pi}{4}\right)\right) \mid \leqslant 1, \text { and } \\
& \mid e^{-\mathrm{i} \sqrt{\mathrm{i} \lambda} \mid}=\exp \left(-\sqrt{|\lambda|} \cos \left(\frac{\theta}{2}-\frac{\pi}{4}\right)\right) \leqslant e^{-\frac{\sqrt{2|\lambda|}}{2}} .
\end{aligned}
$$

Divide the both hand sides of the equation $\Sigma(\lambda)=0$ by $\lambda \sqrt{\mathrm{i} \lambda} e^{\sqrt{\lambda(\lambda+a)}+\sqrt{\mathrm{i} \lambda}+\mathrm{i} \sqrt{\mathrm{i} \lambda}}$, and conduct some routine calculations to obtain

$$
\left(1-e^{-2 \sqrt{\lambda(\lambda+a)}}\right)\left(\mathrm{i}-e^{-2 \sqrt{\mathrm{i} \lambda}}\right)=\mathscr{O}\left(|\lambda|^{-\frac{1}{2}}\right)
$$

as $|\lambda| \rightarrow+\infty$ with $\lambda \in \mathbb{D}_{2}$. Note that the equation $\left(1-e^{-2 \sqrt{\lambda(\lambda+a)}}\right)\left(\mathrm{i}-e^{-2 \sqrt{\mathrm{i} \lambda}}\right)=0$ with the restriction $\lambda \in \mathbb{D}_{2}$ has the solutions

$$
\hat{\lambda}_{1 k}=\frac{-a+\mathrm{i} \sqrt{4 k^{2} \pi^{2}-a^{2}}}{2}
$$


with $k \in \mathbb{N}$, and

$$
\hat{\lambda}_{2 \ell}=\pi^{2}\left(\ell+\frac{1}{4}\right)^{2}
$$

with $\ell \in \mathbb{Z}$. By applying Rouché's theorem (see [15]) and Proposition 2.3, we obtain the spectral asymptotics for $A$ :

$$
\lambda_{1 k}=\frac{-a+\mathrm{i} \sqrt{4 k^{2} \pi^{2}-a^{2}}}{2}+\mathscr{O}\left(k^{-\frac{1}{2}}\right)
$$

as $k \rightarrow \infty$ with $k \in \mathbb{N}$, and $\lambda_{2 \ell}=\pi^{2}\left(\ell+\frac{1}{4}\right)^{2} \mathrm{i}+\mathscr{O}\left(|\ell|^{-\frac{1}{2}}\right)$ as $\ell \rightarrow \infty$ with $\ell \in \mathbb{Z}$.

Finally, since $\lambda \in \sigma(A)$ iff $\bar{\lambda} \in \sigma(A)$, we know that there are eigenvalues of $A$ which are distributed as follows:

$$
\lambda_{1 k}=\frac{-a-\mathrm{i} \sqrt{4 k^{2} \pi^{2}-a^{2}}}{2}+\mathscr{O}\left(k^{-\frac{1}{2}}\right)
$$

as $k \rightarrow \infty$ with $k \in \mathbb{N}$, and $\lambda_{2 \ell}=-\pi^{2}\left(\ell+\frac{1}{4}\right)^{2} \mathrm{i}+\mathscr{O}\left(|\ell|^{-\frac{1}{2}}\right)$ as $\ell \rightarrow \infty$ with $\ell \in \mathbb{Z}$. This, together with the afore-conducted analysis, implies that the proof is complete.

Proof of Theorem 1.1. From Propositions 2.2 and 2.4, we can obtain the desired conclusion immediately.

\section{Proof of Theorem 1.2}

The main objective of this section is to prove that the energy $E(t)$ for system (1.1) defined by (1.4) decreases at a logarithmic decay rate. First, by using the result in $[11,12,13]$, we can deduce energy decreasing property for system (1.1) from Propositions 2.2.

Proposition 3.1. Along every solution $(u, w)$ to system (1.1), $E(t)$ decreases to 0 as $t \rightarrow+\infty$, where $E(t)$ is given by (1.4).

One of our main ingredients in the processes of proving logarithmic energy decay for system (1.1) is to compute Green's functions for the following boundary value problem:

$$
\left.\begin{array}{ll}
\varphi^{\prime \prime}-\left(\lambda^{2}+a \lambda\right) \varphi=f & \text { in }(0,1), \\
\psi^{(4)}+\lambda^{2} \psi=g & \text { in }(0,1), \\
\varphi(0)-\psi(0)=\varphi^{\prime}(0)-\psi^{\prime \prime \prime}(0)=0, & \\
\varphi(1)=\psi(1)=\psi^{\prime \prime}(0)=\psi^{\prime \prime}(1)=0 . &
\end{array}\right\}
$$

Lemma 3.1. Let $\lambda \in \mathrm{i} \mathbb{R}$.

- For every pair $(f, g) \in L^{2}(0,1) \times L^{2}(0,1), B V P(3.1)$ admits a unique solution $(\varphi, \psi) \in H^{2}(0,1) \times$ $H^{4}(0,1)$ such that $(\varphi, 0, \psi, 0) \in \mathscr{D}(A)$.

- There exist four bivariate functions $G^{11}(x, \xi), G^{12}(x, \xi), G^{21}(x, \xi)$, and $G^{22}(x, \xi)$ such that every solution $(\varphi, \psi)$ of BVP (3.1) can be expressed by

$$
\begin{aligned}
& \varphi(x)=\int_{0}^{1} G^{11}(x, \xi) f(\xi) d \xi+\int_{0}^{1} G^{12}(x, \xi) g(\xi) d \xi, \quad \text { and } \\
& \psi(x)=\int_{0}^{1} G^{21}(x, \xi) f(\xi) d \xi+\int_{0}^{1} G^{22}(x, \xi) g(\xi) d \xi .
\end{aligned}
$$


Remark 3.1. The bivariate functions $G^{11}(x, \xi), G^{12}(x, \xi), G^{21}(x, \xi)$, and $G^{22}(x, \xi)$ are so smooth so to qualify all the following calculations, and can be viewed as Green's functions for BVP (3.1).

Proof. The first statement follows immediately from Proposition 2.2. It remains to prove the second statement.

Inspired by the idea of finding Green's function for an boundary value problem for a single ODE, we guess that

$$
\left.\begin{array}{rl}
G^{11}(x, \xi)= & c_{1}^{11} e^{\mu_{1}(x-\xi)}+c_{2}^{11} e^{\mu_{2}(x-\xi)}+H(x-\xi)\left[\check{c}_{1}^{11} e^{\mu_{1}(x-\xi)}+\check{c}_{2}^{11} e^{\mu_{2}(x-\xi)}\right], \\
G^{12}(x, \xi)= & c_{1}^{12} e^{\mu_{1}(x-\xi)}+c_{2}^{12} e^{\mu_{2}(x-\xi)}, \\
G^{21}(x, \xi)= & c_{1}^{21} e^{v_{1}(x-\xi)}+c_{2}^{21} e^{v_{2}(x-\xi)}+c_{3}^{21} e^{v_{3}(x-\xi)}+c_{4}^{21} e^{v_{4}(x-\xi)}, \\
G^{22}(x, \xi)= & c_{1}^{22} e^{v_{1}(x-\xi)}+c_{2}^{22} e^{v_{2}(x-\xi)}+c_{3}^{22} e^{v_{3}(x-\xi)}+c_{4}^{22} e^{v_{4}(x-\xi)} \\
& +H(x-\xi)\left[\check{c}_{1}^{22} e^{v_{1}(x-\xi)}+\check{c}_{2}^{22} e^{v_{2}(x-\xi)}+\check{c}_{3}^{22} e^{v_{3}(x-\xi)}+\check{c}_{4}^{22} e^{v_{4}(x-\xi)}\right],
\end{array}\right\}
$$

where $H$ is the celebrated Heaviside function. $\mu_{i}(i=1,2)$ and $v_{j}(j=1,2,3,4)$ are solutions to the characteristic equations $\mu^{2}-\lambda(\lambda+a)=0$ and $v^{4}-\lambda^{2}=0$, respectively, and are given by

$$
\left.\begin{array}{ll}
\mu_{1}=\sqrt{\lambda^{2}+a \lambda}=\lambda+\frac{a}{2}-\frac{a^{2}}{8 \lambda}+\mathscr{O}\left(|\lambda|^{-2}\right), & \\
\mu_{2}=-\sqrt{\lambda^{2}+a \lambda}=-\lambda-\frac{a}{2}+\frac{a^{2}}{8 \lambda}+\mathscr{O}\left(|\lambda|^{-2}\right), & \\
v_{1}=\sqrt{\lambda}, & v_{2}=-\sqrt{\lambda}, \\
v_{3}=\mathrm{i} \sqrt{\lambda}, & v_{4}=-\mathrm{i} \sqrt{\lambda} .
\end{array}\right\}
$$

$\check{c}_{i}^{11}(i=1,2)$ and $\check{c}_{1}^{22}(j=1,2,3,4)$ are given by

$$
\left.\begin{array}{l}
\check{c}_{1}^{11}=\frac{1}{\mu_{1}-\mu_{2}}=\frac{1}{2 \mu_{1}}, \\
\check{c}_{2}^{11}=\frac{1}{\mu_{2}-\mu_{1}}=-\frac{1}{2 \mu_{1}}
\end{array}\right\}
$$

and

and are determined by

$$
\left.\begin{array}{ll}
\check{c}_{1}^{22}=\frac{1}{4 \lambda^{\frac{3}{2}}}, & \check{c}_{2}^{22}=-\frac{1}{4 \lambda^{\frac{3}{2}}}, \\
\check{c}_{3}^{22}=\frac{i}{4 \lambda^{\frac{3}{2}}}, & \check{c}_{4}^{22}=-\frac{\mathrm{i}}{4 \lambda^{\frac{3}{2}}},
\end{array}\right\}
$$

and

$$
\left.\begin{array}{l}
\check{c}_{1}^{11}+\check{c}_{2}^{11}=0, \\
\mu_{1} \check{c}_{1}^{11}+\mu_{2} \check{c}_{2}^{11}=1
\end{array}\right\}
$$

$$
\left.\begin{array}{l}
\check{c}_{1}^{22}+\check{c}_{2}^{22}+\check{c}_{3}^{22}+\check{c}_{4}^{22}=0, \\
v_{1} \check{c}_{1}^{22}+v_{2} \check{c}_{2}^{22}+v_{3} \check{c}_{3}^{22}+v_{4} \check{c}_{4}^{22}=0, \\
\left(v_{1}\right)^{2} \check{c}_{1}^{22}+\left(v_{2}\right)^{2} \check{c}_{2}^{22}+\left(v_{3}\right)^{2} \check{c}_{3}^{22}+\left(v_{4}\right)^{2} \check{c}_{4}^{22}=0, \\
\left(v_{1}\right)^{3} \check{c}_{1}^{22}+\left(v_{2}\right)^{3} \check{c}_{2}^{22}+\left(v_{3}\right)^{3} \check{c}_{3}^{22}+\left(v_{4}\right)^{3} \check{c}_{4}^{22}=1
\end{array}\right\}
$$


respectively. $c_{i}^{11}, c_{i}^{12}(i=1,2)$ and $c_{j}^{21}, c_{j}^{22}(j=1,2,3,4)$ are yet to be determined shortly. By taking fully use of boundary conditions of BVP (3.1), we have

$$
\begin{aligned}
& \varphi(1)=0, \forall(f, g) \in L^{2}\left(0,1 ; \mathbb{R}^{2}\right) \Longrightarrow G^{11}(1, \xi)=0, G^{12}(1, \xi)=0, \\
& \psi(1)=0, \forall(f, g) \in L^{2}\left(0,1 ; \mathbb{R}^{2}\right) \Longrightarrow G^{21}(1, \xi)=0, G^{22}(1, \xi)=0, \\
& \psi^{\prime \prime}(1)=0, \forall(f, g) \in L^{2}\left(0,1 ; \mathbb{R}^{2}\right) \Longrightarrow \partial_{x}^{2} G^{21}(1, \xi)=0, \partial_{x}^{2} G^{22}(1, \xi)=0 \text {, } \\
& \varphi^{\prime \prime}(0)=0, \forall(f, g) \in L^{2}\left(0,1 ; \mathbb{R}^{2}\right) \Longrightarrow \partial_{x}^{2} G^{21}(0, \xi)=0, \partial_{x}^{2} G^{22}(0, \xi)=0, \\
& \varphi(0)=\psi(0), \forall(f, g) \in L^{2}\left(0,1 ; \mathbb{R}^{2}\right) \Longrightarrow G^{11}(0, \xi)=G^{21}(0, \xi), \\
& G^{12}(0, \xi)=G^{22}(0, \xi), \\
& \varphi^{\prime}(0)=\psi^{\prime \prime \prime}(0), \forall(f, g) \in L^{2}\left(0,1 ; \mathbb{R}^{2}\right) \Longrightarrow \partial_{x} G^{11}(0, \xi)=\partial_{x}^{3} G^{21}(0, \xi), \\
& \partial_{x} G^{12}(0, \xi)=\partial_{x}^{3} G^{22}(0, \xi) \text {. }
\end{aligned}
$$

With the aid of this and (3.2), we have

$$
\begin{aligned}
& G^{11}(1, \xi)=0 \Longrightarrow c_{1}^{11} e^{\mu_{1}(1-\xi)}+c_{2}^{11} e^{\mu_{2}(1-\xi)}=-\check{c}_{1}^{11} e^{\mu_{1}(1-\xi)}-\check{c}_{2}^{11} e^{\mu_{2}(1-\xi)}, \\
& G^{12}(1, \xi)=0 \Longrightarrow c_{1}^{12} e^{\mu_{1}(1-\xi)}+c_{2}^{12} e^{\mu_{2}(1-\xi)}=0, \\
& G^{21}(1, \xi)=0 \Longrightarrow c_{1}^{21} e^{v_{1}(1-\xi)}+c_{2}^{21} e^{v_{2}(1-\xi)}+c_{3}^{21} e^{v_{3}(1-\xi)}+c_{4}^{21} e^{v_{4}(1-\xi)}=0, \\
& G^{22}(1, \xi)=0 \Longrightarrow c_{1}^{22} e^{v_{1}(1-\xi)}+c_{2}^{22} e^{v_{2}(1-\xi)}+c_{3}^{22} e^{v_{3}(1-\xi)}+c_{4}^{22} e^{v_{4}(1-\xi)} \\
& =-\check{c}_{1}^{22} e^{v_{1}(1-\xi)}-\check{c}_{2}^{22} e^{v_{2}(1-\xi)}-\check{c}_{3}^{22} e^{v_{3}(1-\xi)}-\check{c}_{4}^{22} e^{v_{4}(1-\xi)} \\
& \partial_{x}^{2} G^{21}(1, \xi)=0 \Longrightarrow\left(v_{1}\right)^{2} c_{1}^{21} e^{v_{1}(1-\xi)}+\left(v_{2}\right)^{2} c_{2}^{21} e^{v_{2}(1-\xi)}+\left(v_{3}\right)^{2} c_{3}^{21} e^{v_{3}(1-\xi)}+\left(v_{4}\right)^{2} c_{4}^{21} e^{v_{4}(1-\xi)}=0, \\
& \partial_{x}^{2} G^{22}(1, \xi)=0 \Longrightarrow\left(v_{1}\right)^{2} c_{1}^{22} e^{v_{1}(1-\xi)}+\left(v_{2}\right)^{2} c_{2}^{22} e^{v_{2}(1-\xi)}+\left(v_{3}\right)^{2} c_{3}^{22} e^{v_{3}(1-\xi)}+\left(v_{4}\right)^{2} c_{4}^{22} e^{v_{4}(1-\xi)} \\
& =-\left(v_{1}\right)^{2} \check{c}_{1}^{22} e^{v_{1}(1-\xi)}-\left(v_{2}\right)^{2} \check{c}_{2}^{22} e^{v_{2}(1-\xi)}-\left(v_{3}\right)^{2} \check{c}_{3}^{22} e^{v_{3}(1-\xi)}-\left(v_{4}\right)^{2} \check{c}_{4}^{22} e^{v_{4}(1-\xi)}, \\
& \partial_{x}^{2} G^{21}(0, \xi)=0 \Longrightarrow\left(v_{1}\right)^{2} c_{1}^{21} e^{-v_{1} \xi}+\left(v_{2}\right)^{2} c_{2}^{21} e^{-v_{2} \xi}+\left(v_{3}\right)^{2} c_{3}^{21} e^{-v_{3} \xi}+\left(v_{4}\right)^{2} c_{4}^{21} e^{-v_{4} \xi}=0 \text {, } \\
& \partial_{x}^{2} G^{22}(0, \xi)=0 \Longrightarrow\left(v_{1}\right)^{2} c_{1}^{22} e^{-v_{1} \xi}+\left(v_{2}\right)^{2} c_{2}^{22} e^{-v_{2} \xi}+\left(v_{3}\right)^{2} c_{3}^{22} e^{-v_{3} \xi}+\left(v_{4}\right)^{2} c_{4}^{22} e^{-v_{4} \xi}=0 \text {, }
\end{aligned}
$$

and

$$
\left.\begin{array}{rl}
G^{11}(0, \xi)=G^{21}(0, \xi) \Longrightarrow & c_{1}^{21} e^{-v_{1} \xi}+c_{2}^{21} e^{-v_{2} \xi}+c_{3}^{21} e^{-v_{3} \xi}+c_{4}^{21} e^{-v_{4} \xi} \\
& -c_{1}^{11} e^{-\mu_{1} \xi}-c_{2}^{11} e^{-\mu_{2} \xi}=0, \\
G^{12}(0, \xi)=G^{22}(0, \xi) \Longrightarrow & c_{1}^{22} e^{-v_{1} \xi}+c_{2}^{22} e^{-v_{2} \xi}+c_{3}^{22} e^{-v_{3} \xi}+c_{4}^{22} e^{-v_{4} \xi} \\
& -c_{1}^{12} e^{-\mu_{1} \xi}-c_{2}^{12} e^{-\mu_{2} \xi}=0, \\
\partial_{x} G^{11}(0, \xi)=\partial_{x}^{3} G^{21}(0, \xi) \Longrightarrow & \left(v_{1}\right)^{3} c_{1}^{21} e^{-v_{1} \xi}+\left(v_{2}\right)^{3} c_{2}^{21} e^{-v_{2} \xi}+\left(v_{3}\right)^{3} c_{3}^{21} e^{-v_{3} \xi}+\left(v_{4}\right)^{3} c_{4}^{21} e^{-v_{4} \xi} \\
& -\mu_{1} c_{1}^{11} e^{-\mu_{1} \xi}-\mu_{2} c_{2}^{11} e^{-\mu_{2} \xi}=0, \\
\partial_{x} G^{12}(0, \xi)=\partial_{x}^{3} G^{22}(0, \xi) \Longrightarrow & \left(v_{1}\right)^{3} c_{1}^{22} e^{-v_{1} \xi}+\left(v_{2}\right)^{3} c_{2}^{22} e^{-v_{2} \xi}+\left(v_{3}\right)^{3} c_{3}^{22} e^{-v_{3} \xi}+\left(v_{4}\right)^{3} c_{4}^{22} e^{-v_{4} \xi} \\
& -\mu_{1} c_{1}^{12} e^{-\mu_{1} \xi}-\mu_{2} c_{2}^{12} e^{-\mu_{2} \xi}=0 .
\end{array}\right\}
$$


The afore-obtained twelve equations can be regrouped into the following two systems of equations:

$$
\left.\begin{array}{l}
c_{1}^{11} e^{\mu_{1}(1-\xi)}+c_{2}^{11} e^{\mu_{2}(1-\xi)}=-\check{c}_{1}^{11} e^{\mu_{1}(1-\xi)}-\check{c}_{2}^{11} e^{\mu_{2}(1-\xi)}, \\
c_{1}^{21} e^{v_{1}(1-\xi)}+c_{2}^{21} e^{v_{2}(1-\xi)}+c_{3}^{21} e^{v_{3}(1-\xi)}+c_{4}^{21} e^{v_{4}(1-\xi)}=0, \\
\left(v_{1}\right)^{2} c_{1}^{21} e^{v_{1}(1-\xi)}+\left(v_{2}\right)^{2} c_{2}^{21} e^{v_{2}(1-\xi)}+\left(v_{3}\right)^{2} c_{3}^{21} e^{v_{3}(1-\xi)}+\left(v_{4}\right)^{2} c_{4}^{21} e^{v_{4}(1-\xi)}=0, \\
\left(v_{1}\right)^{2} c_{1}^{21} e^{-v_{1} \xi}+\left(v_{2}\right)^{2} c_{2}^{21} e^{-v_{2} \xi}+\left(v_{3}\right)^{2} c_{3}^{21} e^{-v_{3} \xi}+\left(v_{4}\right)^{2} c_{4}^{21} e^{-v_{4} \xi}=0, \\
c_{1}^{21} e^{-v_{1} \xi}+c_{2}^{21} e^{-v_{2} \xi}+c_{3}^{21} e^{-v_{3} \xi}+c_{4}^{21} e^{-v_{4} \xi}-c_{1}^{11} e^{-\mu_{1} \xi}-c_{2}^{11} e^{-\mu_{2} \xi}=0, \\
\left(v_{1}\right)^{3} c_{1}^{21} e^{-v_{1} \xi}+\left(v_{2}\right)^{3} c_{2}^{21} e^{-v_{2} \xi}+\left(v_{3}\right)^{3} c_{3}^{21} e^{-v_{3} \xi}+\left(v_{4}\right)^{3} c_{4}^{21} e^{-v_{4} \xi}-\mu_{1} c_{1}^{11} e^{-\mu_{1} \xi}-\mu_{2} c_{2}^{11} e^{-\mu_{2} \xi}=0,
\end{array}\right\}
$$

and

$$
\left.\begin{array}{l}
c_{1}^{12} e^{\mu_{1}(1-\xi)}+c_{2}^{12} e^{\mu_{2}(1-\xi)}=0, \\
c_{1}^{22} e^{v_{1}(1-\xi)}+c_{2}^{22} e^{v_{2}(1-\xi)}+c_{3}^{22} e^{v_{3}(1-\xi)}+c_{4}^{22} e^{v_{4}(1-\xi)} \\
=-\check{c}_{1}^{22} e^{v_{1}(1-\xi)}-\check{c}_{2}^{22} e^{v_{2}(1-\xi)}-\breve{c}_{3}^{22} e^{v_{3}(1-\xi)}-\breve{c}_{4}^{22} e^{v_{4}(1-\xi)}, \\
\left(v_{1}\right)^{2} c_{1}^{22} e^{v_{1}(1-\xi)}+\left(v_{2}\right)^{2} c_{2}^{22} e^{v_{2}(1-\xi)}+\left(v_{3}\right)^{2} c_{3}^{22} e^{v_{3}(1-\xi)}+\left(v_{4}\right)^{2} c_{4}^{22} e^{v_{4}(1-\xi)} \\
=-\left(v_{1}\right)^{2} \check{c}_{1}^{22} e^{v_{1}(1-\xi)}-\left(v_{2}\right)^{2} \check{c}_{2}^{22} e^{v_{2}(1-\xi)}-\left(v_{3}\right)^{2} \check{c}_{3}^{22} e^{v_{3}(1-\xi)}-\left(v_{4}\right)^{2} \check{c}_{4}^{22} e^{v_{4}(1-\xi)}, \\
\left(v_{1}\right)^{2} c_{1}^{22} e^{-v_{1} \xi}+\left(v_{2}\right)^{2} c_{2}^{22} e^{-v_{2} \xi}+\left(v_{3}\right)^{2} c_{3}^{22} e^{-v_{3} \xi}+\left(v_{4}\right)^{2} c_{4}^{22} e^{-v_{4} \xi}=0, \\
c_{1}^{22} e^{-v_{1} \xi}+c_{2}^{22} e^{-v_{2} \xi}+c_{3}^{22} e^{-v_{3} \xi}+c_{4}^{22} e^{-v_{4} \xi}-c_{1}^{12} e^{-\mu_{1} \xi}-c_{2}^{12} e^{-\mu_{2} \xi}=0, \\
\left(v_{1}\right)^{3} c_{1}^{22} e^{-v_{1} \xi}+\left(v_{2}\right)^{3} c_{2}^{22} e^{-v_{2} \xi}+\left(v_{3}\right)^{3} c_{3}^{22} e^{-v_{3} \xi}+\left(v_{4}\right)^{3} c_{4}^{22} e^{-v_{4} \xi} \\
-\mu_{1} c_{1}^{12} e^{-\mu_{1} \xi}-\mu_{2} c_{2}^{12} e^{-\mu_{2} \xi}=0 .
\end{array}\right\}
$$

These two systems, together with Carmer's rule, imply

$$
\left.\begin{array}{ll}
c_{1}^{11}=\frac{\Delta_{1}^{f}}{\Delta}, & c_{2}^{11}=\frac{\Delta_{2}^{f}}{\Delta}, \\
c_{1}^{21}=\frac{\Delta_{3}^{f}}{\Delta}, & c_{2}^{21}=\frac{\Delta_{4}^{f}}{\Delta}, \quad c_{3}^{21}=\frac{\Delta_{5}^{f}}{\Delta}, \quad c_{4}^{21}=\frac{\Delta_{6}^{f}}{\Delta}, \\
c_{1}^{12}=\frac{\Delta_{1}^{g}}{\Delta}, & c_{2}^{12}=\frac{\Delta_{2}^{g}}{\Delta}, \\
c_{1}^{22}=\frac{\Delta_{3}^{g}}{\Delta}, & c_{2}^{22}=\frac{\Delta_{4}^{g}}{\Delta}, \quad c_{3}^{22}=\frac{\Delta_{5}^{g}}{\Delta}, \quad c_{4}^{22}=\frac{\Delta_{6}^{g}}{\Delta},
\end{array}\right\}
$$

where

$$
\begin{aligned}
\Delta & =e^{-\left(\mu_{1}+\mu_{2}+\sum_{k=1}^{4} v_{k}\right) \xi} \operatorname{det}\left(\begin{array}{cccccc}
e^{\mu_{1}} & e^{\mu_{2}} & 0 & 0 & 0 & 0 \\
0 & 0 & e^{v_{1}} & e^{v_{2}} & e^{v_{3}} & e^{v_{4}} \\
0 & 0 & \left(v_{1}\right)^{2} e^{v_{1}} & \left(v_{2}\right)^{2} e^{v_{2}} & \left(v_{3}\right)^{2} e^{v_{3}} & \left(v_{4}\right)^{2} e^{v_{4}} \\
0 & 0 & \left(v_{1}\right)^{2} & \left(v_{2}\right)^{2} & \left(v_{3}\right)^{2} & \left(v_{4}\right)^{2} \\
-1 & -1 & 1 & 1 & 1 & 1 \\
-\mu_{1} & -\mu_{2} & \left(v_{1}\right)^{3} & \left(v_{2}\right)^{3} & \left(v_{3}\right)^{3} & \left(v_{4}\right)^{3}
\end{array}\right) \\
= & 2(1+\mathrm{i}) \lambda^{\frac{7}{2}}\left(e^{\mu_{1}}-e^{\mu_{2}}\right)\left[e^{(1-\mathrm{i}) \sqrt{\lambda}}+\mathrm{i} e^{(1+\mathrm{i}) \sqrt{\lambda}}-\mathrm{i} e^{-(1+\mathrm{i}) \sqrt{\lambda}}-e^{(-1+\mathrm{i}) \sqrt{\lambda}}\right] \\
& +4 \lambda^{2}\left(\mu_{1} e^{\mu_{2}}-\mu_{2} e^{\mu_{1}}\right)\left[-e^{(1-\mathrm{i}) \sqrt{\lambda}}+e^{(1+\mathrm{i}) \sqrt{\lambda}}+e^{-(1+\mathrm{i}) \sqrt{\lambda}}-e^{(-1+\mathrm{i}) \sqrt{\lambda}}\right],
\end{aligned}
$$




$$
\begin{aligned}
\Delta_{1}^{f}= & -\left(\check{c}_{1}^{11} e^{\mu_{1}(1-\xi)}+\check{c}_{2}^{11} e^{\mu_{2}(1-\xi)}\right) \\
& \times \operatorname{det}\left(\begin{array}{ccccc}
0 & e^{v_{1}(1-\xi)} & e^{v_{2}(1-\xi)} & e^{v_{3}(1-\xi)} & e^{v_{4}(1-\xi)} \\
0 & \left(v_{1}\right)^{2} e^{v_{1}(1-\xi)} & \left(v_{2}\right)^{2} e^{v_{2}(1-\xi)} & \left(v_{3}\right)^{2} e^{v_{3}(1-\xi)} & \left(v_{4}\right)^{2} e^{v_{4}(1-\xi)} \\
0 & \left(v_{1}\right)^{2} e^{-v_{1} \xi} & \left(v_{2}\right)^{2} e^{-v_{2} \xi} & \left(v_{3}\right)^{2} e^{-v_{3} \xi} & \left(v_{4}\right)^{2} e^{-v_{4} \xi} \\
-e^{-\mu_{2} \xi} & e^{-v_{1} \xi} & e^{-v_{2} \xi} & e^{-v_{3} \xi} & e^{-v_{4} \xi} \\
-\mu_{2} e^{-\mu_{2} \xi} & \left(v_{1}\right)^{3} e^{-v_{1} \xi} & \left(v_{2}\right)^{3} e^{-v_{2} \xi} & \left(v_{3}\right)^{3} e^{-v_{3} \xi} & \left(v_{4}\right)^{3} e^{-v_{4} \xi}
\end{array}\right) \\
= & 4 \lambda^{2} \mu_{2}\left(\check{c}_{1}^{11} e^{\mu_{1}}+\check{c}_{2}^{11} e^{\mu_{2}(1-2 \xi)}\right)\left[-e^{(1-\mathrm{i}) \sqrt{\lambda}}+e^{(1+\mathrm{i}) \sqrt{\lambda}}+e^{-(1+\mathrm{i}) \sqrt{\lambda}}-e^{(-1+\mathrm{i}) \sqrt{\lambda}}\right] \\
& -2 \lambda^{\frac{7}{2}}(1+\mathrm{i})\left(\check{c}_{1}^{11} e^{\mu_{1}}+\check{c}_{2}^{11} e^{\mu_{2}(1-2 \xi)}\right)\left[e^{(1-\mathrm{i}) \sqrt{\lambda}}+\mathrm{i} e^{(1+\mathrm{i}) \sqrt{\lambda}}-\mathrm{i} e^{-(1+\mathrm{i}) \sqrt{\lambda}}-e^{(-1+\mathrm{i}) \sqrt{\lambda}}\right],
\end{aligned}
$$

$$
\begin{aligned}
\Delta_{2}^{f}= & \left(\check{c}_{1}^{11} e^{\mu_{1}(1-\xi)}+\check{c}_{2}^{11} e^{\mu_{2}(1-\xi)}\right) \\
& \times \operatorname{det}\left(\begin{array}{ccccc}
0 & e^{v_{1}(1-\xi)} & e^{v_{2}(1-\xi)} & e^{v_{3}(1-\xi)} & e^{v_{4}(1-\xi)} \\
0 & \left(v_{1}\right)^{2} e^{v_{1}(1-\xi)} & \left(v_{2}\right)^{2} e^{v_{2}(1-\xi)} & \left(v_{3}\right)^{2} e^{v_{3}(1-\xi)} & \left(v_{4}\right)^{2} e^{v_{4}(1-\xi)} \\
0 & \left(v_{1}\right)^{2} e^{-v_{1} \xi} & \left(v_{2}\right)^{2} e^{-v_{2} \xi} & \left(v_{3}\right)^{2} e^{-v_{3} \xi} & \left(v_{4}\right)^{2} e^{-v_{4} \xi} \\
-e^{-\mu_{1} \xi} & e^{-v_{1} \xi} & e^{-v_{2} \xi} & e^{-v_{3} \xi} & e^{-v_{4} \xi} \\
-\mu_{1} e^{-\mu_{1} \xi} & \left(v_{1}\right)^{3} e^{-v_{1} \xi} & \left(v_{2}\right)^{3} e^{-v_{2} \xi} & \left(v_{3}\right)^{3} e^{-v_{3} \xi} & \left(v_{4}\right)^{3} e^{-v_{4} \xi}
\end{array}\right) \\
= & 2 \lambda^{\frac{7}{2}}(1+\mathrm{i})\left(\check{c}_{1}^{11} e^{\mu_{1}(1-2 \xi)}+\check{c}_{2}^{11} e^{\mu_{2}}\right)\left[e^{(1-\mathrm{i}) \sqrt{\lambda}}+\mathrm{i} e^{(1+\mathrm{i}) \sqrt{\lambda}}-\mathrm{i} e^{-(1+\mathrm{i}) \sqrt{\lambda}}-e^{(-1+\mathrm{i}) \sqrt{\lambda}}\right] \\
& -4 \lambda^{2} \mu_{1}\left(\check{c}_{1}^{11} e^{\mu_{1}(1-2 \xi)}+\check{c}_{2}^{11} e^{\mu_{2}}\right)\left[-e^{(1-\mathrm{i}) \sqrt{\lambda}}+e^{(1+\mathrm{i}) \sqrt{\lambda}}+e^{-(1+\mathrm{i}) \sqrt{\lambda}}-e^{(-1+\mathrm{i}) \sqrt{\lambda}}\right]
\end{aligned}
$$

$$
\begin{aligned}
\Delta_{3}^{f}= & -\left(\check{c}_{1}^{11} e^{\mu_{1}(1-\xi)}+\check{c}_{2}^{11} e^{\mu_{2}(1-\xi)}\right) \\
& \times \operatorname{det}\left(\begin{array}{ccccc}
0 & 0 & e^{v_{2}(1-\xi)} & e^{v_{3}(1-\xi)} & e^{v_{4}(1-\xi)} \\
0 & 0 & \left(v_{2}\right)^{2} e^{v_{2}(1-\xi)} & \left(v_{3}\right)^{2} e^{v_{3}(1-\xi)} & \left(v_{4}\right)^{2} e^{v_{4}(1-\xi)} \\
0 & 0 & \left(v_{2}\right)^{2} e^{-v_{2} \xi} & \left(v_{3}\right)^{2} e^{-v_{3} \xi} & \left(v_{4}\right)^{2} e^{-v_{4} \xi} \\
-e^{-\mu_{1} \xi} & -e^{-\mu_{2} \xi} & e^{-v_{2} \xi} & e^{-v_{3} \xi} & e^{-v_{4} \xi} \\
-\mu_{1} e^{-\mu_{1} \xi} & -\mu_{2} e^{-\mu_{2} \xi} & \left(v_{2}\right)^{3} e^{-v_{2} \xi} & \left(v_{3}\right)^{3} e^{-v_{3} \xi} & \left(v_{4}\right)^{3} e^{-v_{4} \xi}
\end{array}\right) \\
& =2 \lambda^{2} e^{\sqrt{\lambda} \xi}\left(\mu_{1}-\mu_{2}\right)\left(\check{c}_{1}^{11} e^{\mu_{1}(1-\xi)}+\check{c}_{2}^{11} e^{\mu_{2}(1-\xi)}\right)\left[e^{(-1+\mathrm{i}) \sqrt{\lambda}}-e^{-(1+\mathrm{i}) \sqrt{\lambda}}\right],
\end{aligned}
$$

$$
\begin{aligned}
\Delta_{4}^{f}= & -\left(\check{c}_{1}^{11} e^{\mu_{1}(1-\xi)}+\check{c}_{2}^{11} e^{\mu_{2}(1-\xi)}\right) \\
& \times \operatorname{det}\left(\begin{array}{ccccc}
0 & 0 & e^{v_{1}(1-\xi)} & e^{v_{3}(1-\xi)} & e^{v_{4}(1-\xi)} \\
0 & 0 & \left(v_{1}\right)^{2} e^{v_{1}(1-\xi)} & \left(v_{3}\right)^{2} e^{v_{3}(1-\xi)} & \left(v_{4}\right)^{2} e^{v_{4}(1-\xi)} \\
0 & 0 & \left(v_{1}\right)^{2} e^{-v_{1} \xi} & \left(v_{3}\right)^{2} e^{-v_{3} \xi} & \left(v_{4}\right)^{2} e^{-v_{4} \xi} \\
-e^{-\mu_{1} \xi} & -e^{-\mu_{2} \xi} & e^{-v_{1} \xi} & e^{-v_{3} \xi} & e^{-v_{4} \xi} \\
-\mu_{1} e^{-\mu_{1} \xi} & -\mu_{2} e^{-\mu_{2} \xi} & \left(v_{1}\right)^{3} e^{-v_{1} \xi} & \left(v_{3}\right)^{3} e^{-v_{3} \xi} & \left(v_{4}\right)^{3} e^{-v_{4} \xi}
\end{array}\right) \\
& =2 \lambda^{2} e^{-\sqrt{\lambda} \xi}\left(\mu_{1}-\mu_{2}\right)\left(\check{c}_{1}^{11} e^{\mu_{1}(1-\xi)}+\check{c}_{2}^{11} e^{\mu_{2}(1-\xi)}\right)\left[e^{(1+\mathrm{i}) \sqrt{\lambda}}-e^{(1-\mathrm{i}) \sqrt{\lambda}}\right],
\end{aligned}
$$




$$
\begin{aligned}
\Delta_{5}^{f}= & -\left(\check{c}_{1}^{11} e^{\mu_{1}(1-\xi)}+\check{c}_{2}^{11} e^{\mu_{2}(1-\xi)}\right) \\
& \times \operatorname{det}\left(\begin{array}{ccccc}
0 & 0 & e^{v_{1}(1-\xi)} & e^{v_{2}(1-\xi)} & e^{v_{4}(1-\xi)} \\
0 & 0 & \left(v_{1}\right)^{2} e^{v_{1}(1-\xi)} & \left(v_{2}\right)^{2} e^{v_{2}(1-\xi)} & \left(v_{4}\right)^{2} e^{v_{4}(1-\xi)} \\
0 & 0 & \left(v_{1}\right)^{2} e^{-v_{1} \xi} & \left(v_{2}\right)^{2} e^{-v_{2} \xi} & \left(v_{4}\right)^{2} e^{-v_{4} \xi} \\
-e^{-\mu_{1} \xi} & -e^{-\mu_{2} \xi} & e^{-v_{1} \xi} & e^{-v_{2} \xi} & e^{-v_{4} \xi} \\
-\mu_{1} e^{-\mu_{1} \xi} & -\mu_{2} e^{-\mu_{2} \xi} & \left(v_{1}\right)^{3} e^{-v_{1} \xi} & \left(v_{2}\right)^{3} e^{-v_{2} \xi} & \left(v_{4}\right)^{3} e^{-v_{4} \xi}
\end{array}\right) \\
= & 2 \lambda^{2} e^{\mathrm{i} \sqrt{\lambda \xi}}\left(\mu_{1}-\mu_{2}\right)\left(\check{c}_{1}^{11} e^{\mu_{1}(1-\xi)}+\check{c}_{2}^{11} e^{\mu_{2}(1-\xi)}\right)\left[e^{(1-\mathrm{i}) \sqrt{\lambda}}-e^{-(1+\mathrm{i}) \sqrt{\lambda}}\right],
\end{aligned}
$$

$$
\begin{aligned}
\Delta_{6}^{f}= & -\left(\check{c}_{1}^{11} e^{\mu_{1}(1-\xi)}+\check{c}_{2}^{11} e^{\mu_{2}(1-\xi)}\right) \\
& \times \operatorname{det}\left(\begin{array}{ccccc}
0 & 0 & e^{v_{1}(1-\xi)} & e^{v_{2}(1-\xi)} & e^{v_{3}(1-\xi)} \\
0 & 0 & \left(v_{1}\right)^{2} e^{v_{1}(1-\xi)} & \left(v_{2}\right)^{2} e^{v_{2}(1-\xi)} & \left(v_{3}\right)^{2} e^{v_{3}(1-\xi)} \\
0 & 0 & \left(v_{1}\right)^{2} e^{-v_{1} \xi} & \left(v_{2}\right)^{2} e^{-v_{2} \xi} & \left(v_{3}\right)^{2} e^{-v_{3} \xi} \\
-e^{-\mu_{1} \xi} & -e^{-\mu_{2} \xi} & e^{-v_{1} \xi} & e^{-v_{2} \xi} & e^{-v_{3} \xi} \\
-\mu_{1} e^{-\mu_{1} \xi} & -\mu_{2} e^{-\mu_{2} \xi} & \left(v_{1}\right)^{3} e^{-v_{1} \xi} & \left(v_{2}\right)^{3} e^{-v_{2} \xi} & \left(v_{3}\right)^{3} e^{-v_{3} \xi}
\end{array}\right) \\
= & 2 \lambda^{2} e^{-\mathrm{i} \sqrt{\lambda} \xi}\left(\mu_{1}-\mu_{2}\right)\left(\check{c}_{1}^{11} e^{\mu_{1}(1-\xi)}+\check{c}_{2}^{11} e^{\mu_{2}(1-\xi)}\right)\left[e^{(1-\mathrm{i}) \sqrt{\lambda}}-e^{(-1+\mathrm{i}) \sqrt{\lambda}}\right],
\end{aligned}
$$

$$
\begin{aligned}
& \Delta_{1}^{g}=\left(\check{c}_{1}^{22} e^{v_{1}(1-\xi)}+\check{c}_{2}^{22} e^{v_{2}(1-\xi)}+\check{c}_{3}^{22} e^{v_{3}(1-\xi)}+\check{c}_{4}^{22} e^{v_{4}(1-\xi)}\right) \\
& \times \operatorname{det}\left(\begin{array}{ccccc}
e^{\mu_{2}(1-\xi)} & 0 & 0 & 0 & 0 \\
0 & \left(v_{1}\right)^{2} e^{v_{1}(1-\xi)} & \left(v_{2}\right)^{2} e^{v_{2}(1-\xi)} & \left(v_{3}\right)^{2} e^{v_{3}(1-\xi)} & \left(v_{4}\right)^{2} e^{v_{4}(1-\xi)} \\
0 & \left(v_{1}\right)^{2} e^{-v_{1} \xi} & \left(v_{2}\right)^{2} e^{-v_{2} \xi} & \left(v_{3}\right)^{2} e^{-v_{3} \xi} & \left(v_{4}\right)^{2} e^{-v_{4} \xi} \\
-e^{-\mu_{2} \xi} & e^{-v_{1} \xi} & e^{-v_{2} \xi} & e^{-v_{3} \xi} & e^{-v_{4} \xi} \\
-\mu_{2} e^{-\mu_{2} \xi} & \left(v_{1}\right)^{3} e^{-v_{1} \xi} & \left(v_{2}\right)^{3} e^{-v_{2} \xi} & \left(v_{3}\right)^{3} e^{-v_{3} \xi} & \left(v_{4}\right)^{3} e^{-v_{4} \xi}
\end{array}\right) \\
& -\left(\left(v_{1}\right)^{2} \check{c}_{1}^{22} e^{v_{1}(1-\xi)}+\left(v_{2}\right)^{2} \check{c}_{2}^{22} e^{v_{2}(1-\xi)}+\left(v_{3}\right)^{2} \check{c}_{3}^{22} e^{v_{3}(1-\xi)}+\left(v_{4}\right)^{2} \breve{c}_{4}^{22} e^{v_{4}(1-\xi)}\right) \\
& \times \operatorname{det}\left(\begin{array}{ccccc}
e^{\mu_{2}(1-\xi)} & 0 & 0 & 0 & 0 \\
0 & e^{v_{1}(1-\xi)} & e^{v_{2}(1-\xi)} & e^{v_{3}(1-\xi)} & e^{v_{4}(1-\xi)} \\
0 & \left(v_{1}\right)^{2} e^{-v_{1} \xi} & \left(v_{2}\right)^{2} e^{-v_{2} \xi} & \left(v_{3}\right)^{2} e^{-v_{3} \xi} & \left(v_{4}\right)^{2} e^{-v_{4} \xi} \\
-e^{-\mu_{2} \xi} & e^{-v_{1} \xi} & e^{-v_{2} \xi} & e^{-v_{3} \xi} & e^{-v_{4} \xi} \\
-\mu_{2} e^{-\mu_{2} \xi} & \left(v_{1}\right)^{3} e^{-v_{1} \xi} & \left(v_{2}\right)^{3} e^{-v_{2} \xi} & \left(v_{3}\right)^{3} e^{-v_{3} \xi} & \left(v_{4}\right)^{3} e^{-v_{4} \xi}
\end{array}\right) \\
& =4 \lambda^{\frac{7}{2}} e^{\mu_{2}(1-\xi)}\left(e^{v_{4}}+\mathrm{i} e^{v_{1}}-e^{v_{3}}-\mathrm{i} e^{v_{2}}\right)\left[\check{c}_{1}^{22} e^{v_{1}(1-\xi)}+\check{c}_{2}^{22} e^{v_{2}(1-\xi)}+\check{c}_{3}^{22} e^{v_{3}(1-\xi)}+\check{c}_{4}^{22} e^{v_{4}(1-\xi)}\right] \\
& -4 \lambda^{\frac{5}{2}} e^{\mu_{2}(1-\xi)}\left(e^{v_{4}}+\mathrm{i} e^{v_{1}}-e^{v_{3}}-\mathrm{i} e^{v_{2}}\right)\left[\left(v_{1}\right)^{2} \check{c}_{1}^{22} e^{v_{1}(1-\xi)}+\left(v_{2}\right)^{2} \check{c}_{2}^{22} e^{v_{2}(1-\xi)}+\left(v_{3}\right)^{2} \check{c}_{3}^{22} e^{\nu_{3}(1-\xi)}\right. \\
& \left.+\left(v_{4}\right)^{2} \check{c}_{4}^{22} e^{v_{4}(1-\xi)}\right]
\end{aligned}
$$




$$
\begin{aligned}
& \Delta_{2}^{g}=-\left[\check{c}_{1}^{22} e^{v_{1}(1-\xi)}+\check{c}_{2}^{22} e^{v_{2}(1-\xi)}+\check{c}_{3}^{22} e^{v_{3}(1-\xi)}+\check{c}_{4}^{22} e^{v_{4}(1-\xi)}\right] \\
& \times \operatorname{det}\left(\begin{array}{ccccc}
e^{\mu_{1}(1-\xi)} & 0 & 0 & 0 & 0 \\
0 & \left(v_{1}\right)^{2} e^{v_{1}(1-\xi)} & \left(v_{2}\right)^{2} e^{v_{2}(1-\xi)} & \left(v_{3}\right)^{2} e^{v_{3}(1-\xi)} & \left(v_{4}\right)^{2} e^{v_{4}(1-\xi)} \\
0 & \left(v_{1}\right)^{2} e^{-v_{1} \xi} & \left(v_{2}\right)^{2} e^{-v_{2} \xi} & \left(v_{3}\right)^{2} e^{-v_{3} \xi} & \left(v_{4}\right)^{2} e^{-v_{4} \xi} \\
-e^{-\mu_{1} \xi} & e^{-v_{1} \xi} & e^{-v_{2} \xi} & e^{-v_{3} \xi} & e^{-v_{4} \xi} \\
-\mu_{1} e^{-\mu_{1} \xi} & \left(v_{1}\right)^{3} e^{-v_{1} \xi} & \left(v_{2}\right)^{3} e^{-v_{2} \xi} & \left(v_{3}\right)^{3} e^{-v_{3} \xi} & \left(v_{4}\right)^{3} e^{-v_{4} \xi}
\end{array}\right) \\
& +\left[\left(v_{1}\right)^{2} \breve{c}_{1}^{22} e^{v_{1}(1-\xi)}+\left(v_{2}\right)^{2} \breve{c}_{2}^{22} e^{v_{2}(1-\xi)}+\left(v_{3}\right)^{2} \check{c}_{3}^{22} e^{v_{3}(1-\xi)}+\left(v_{4}\right)^{2} \breve{c}_{4}^{22} e^{v_{4}(1-\xi)}\right] \\
& \times \operatorname{det}\left(\begin{array}{ccccc}
e^{\mu_{1}(1-\xi)} & 0 & 0 & 0 & 0 \\
0 & e^{v_{1}(1-\xi)} & e^{v_{2}(1-\xi)} & e^{v_{3}(1-\xi)} & e^{v_{4}(1-\xi)} \\
0 & \left(v_{1}\right)^{2} e^{-v_{1} \xi} & \left(v_{2}\right)^{2} e^{-v_{2} \xi} & \left(v_{3}\right)^{2} e^{-v_{3} \xi} & \left(v_{4}\right)^{2} e^{-v_{4} \xi} \\
-e^{-\mu_{1} \xi} & e^{-v_{1} \xi} & e^{-v_{2} \xi} & e^{-v_{3} \xi} & e^{-v_{4} \xi} \\
-\mu_{1} e^{-\mu_{1} \xi} & \left(v_{1}\right)^{3} e^{-v_{1} \xi} & \left(v_{2}\right)^{3} e^{-v_{2} \xi} & \left(v_{3}\right)^{3} e^{-v_{3} \xi} & \left(v_{4}\right)^{3} e^{-v_{4} \xi}
\end{array}\right) \\
& =-4 \lambda^{\frac{7}{2}} e^{\mu_{1}(1-\xi)}\left(e^{v_{4}}+\mathrm{i} e^{v_{1}}-e^{\nu_{3}}-\mathrm{i} e^{v_{2}}\right)\left[\check{c}_{1}^{22} e^{v_{1}(1-\xi)}+\check{c}_{2}^{22} e^{v_{2}(1-\xi)}+\check{c}_{3}^{22} e^{v_{3}(1-\xi)}+\check{c}_{4}^{22} e^{v_{4}(1-\xi)}\right] \\
& +4 \lambda^{\frac{5}{2}} e^{\mu_{1}(1-\xi)}\left(e^{v_{4}}+\mathrm{i} e^{v_{1}}-e^{v_{3}}-\mathrm{i} e^{v_{2}}\right)\left[\left(v_{1}\right)^{2} \check{c}_{1}^{22} e^{v_{1}(1-\xi)}+\left(v_{2}\right)^{2} \check{c}_{2}^{22} e^{v_{2}(1-\xi)}+\left(v_{3}\right)^{2} \check{c}_{3}^{22} e^{v_{3}(1-\xi)}\right. \\
& \left.+\left(v_{4}\right)^{2} \check{c}_{4}^{22} e^{v_{4}(1-\xi)}\right]
\end{aligned}
$$

$$
\begin{aligned}
& \Delta_{3}^{g}=\left(\check{c}_{1}^{22} e^{v_{1}(1-\xi)}+\check{c}_{2}^{22} e^{\nu_{2}(1-\xi)}+\check{c}_{3}^{22} e^{\nu_{3}(1-\xi)}+\check{c}_{4}^{22} e^{v_{4}(1-\xi)}\right) \\
& \times \operatorname{det}\left(\begin{array}{ccccc}
e^{\mu_{1}(1-\xi)} & e^{\mu_{2}(1-\xi)} & 0 & 0 & 0 \\
0 & 0 & \left(v_{2}\right)^{2} e^{v_{2}(1-\xi)} & \left(v_{3}\right)^{2} e^{v_{3}(1-\xi)} & \left(v_{4}\right)^{2} e^{v_{4}(1-\xi)} \\
0 & 0 & \left(v_{2}\right)^{2} e^{-v_{2} \xi} & \left(v_{3}\right)^{2} e^{-v_{3} \xi} & \left(v_{4}\right)^{2} e^{-v_{4} \xi} \\
-e^{-\mu_{1} \xi} & -e^{-\mu_{2} \xi} & e^{-v_{2} \xi} & e^{-v_{3} \xi} & e^{-v_{4} \xi} \\
-\mu_{1} e^{-\mu_{1} \xi} & -\mu_{2} e^{-\mu_{2} \xi} & \left(v_{2}\right)^{3} e^{-v_{2} \xi} & \left(v_{3}\right)^{3} e^{-v_{3} \xi} & \left(v_{4}\right)^{3} e^{-v_{4} \xi}
\end{array}\right) \\
& -\left(\left(v_{1}\right)^{2} \check{c}_{1}^{22} e^{v_{1}(1-\xi)}+\left(v_{2}\right)^{2} \check{c}_{2}^{22} e^{v_{2}(1-\xi)}+\left(v_{3}\right)^{2} \check{c}_{3}^{22} e^{v_{3}(1-\xi)}+\left(v_{4}\right)^{2} \check{c}_{4}^{22} e^{v_{4}(1-\xi)}\right) \\
& \times \operatorname{det}\left(\begin{array}{ccccc}
e^{\mu_{1}(1-\xi)} & e^{\mu_{2}(1-\xi)} & 0 & 0 & 0 \\
0 & 0 & e^{v_{2}(1-\xi)} & e^{v_{3}(1-\xi)} & e^{v_{4}(1-\xi)} \\
0 & 0 & \left(v_{2}\right)^{2} e^{-v_{2} \xi} & \left(v_{3}\right)^{2} e^{-v_{3} \xi} & \left(v_{4}\right)^{2} e^{-v_{4} \xi} \\
-e^{-\mu_{1} \xi} & -e^{-\mu_{2} \xi} & e^{-v_{2} \xi} & e^{-v_{3} \xi} & e^{-v_{4} \xi} \\
-\mu_{1} e^{-\mu_{1} \xi} & -\mu_{2} e^{-\mu_{2} \xi} & \left(v_{2}\right)^{3} e^{-v_{2} \xi} & \left(v_{3}\right)^{3} e^{-v_{3} \xi} & \left(v_{4}\right)^{3} e^{-v_{4} \xi}
\end{array}\right) \\
& =e^{-v_{2} \xi}\left[\check{c}_{1}^{22} e^{v_{1}(1-\xi)}+\check{c}_{2}^{22} e^{v_{2}(1-\xi)}+\check{c}_{3}^{22} e^{v_{3}(1-\xi)}+\check{c}_{4}^{22} e^{v_{4}(1-\xi)}\right] \\
& \times\left[\lambda^{\frac{7}{2}}\left(e^{\mu_{2}}-e^{\mu_{1}}\right)\left((\mathrm{i}-1) e^{\mathrm{i} \sqrt{\lambda}}+(1+\mathrm{i}) e^{-\mathrm{i} \sqrt{\lambda}}-2 \mathrm{i} e^{-\sqrt{\lambda}}\right)-2 \lambda^{2}\left(\mu_{1} e^{\mu_{2}}-\mu_{2} e^{\mu_{1}}\right)\left(e^{\mathrm{i} \sqrt{\lambda}}-e^{-\mathrm{i} \sqrt{\lambda}}\right)\right] \\
& -e^{-v_{2} \xi}\left[\left(v_{1}\right)^{2} \check{c}_{1}^{22} e^{v_{1}(1-\xi)}+\left(v_{2}\right)^{2} \check{c}_{2}^{22} e^{v_{2}(1-\xi)}+\left(v_{3}\right)^{2} \check{c}_{3}^{22} e^{v_{3}(1-\xi)}+\left(v_{4}\right)^{2} \check{c}_{4}^{22} e^{v_{4}(1-\xi)}\right] \\
& \times\left[\lambda^{\frac{5}{2}}\left(e^{\mu_{2}}-e^{\mu_{1}}\right)\left((1-\mathrm{i}) e^{\mathrm{i} \sqrt{\lambda}}-2 \mathrm{i} e^{-\sqrt{\lambda}}-(1+\mathrm{i}) e^{-\mathrm{i} \sqrt{\lambda}}\right)\right. \\
& \left.-2 \lambda\left(\mu_{1} e^{\mu_{2}}-\mu_{2} e^{\mu_{1}}\right)\left(e^{-\mathrm{i} \sqrt{\lambda}}-e^{\mathrm{i} \sqrt{\lambda}}\right)\right],
\end{aligned}
$$


A LARGE TIME BEHAVIOR PROBLEM FOR A COUPLED SYSTEM OF 1-D WAVE AND PLATE EQUATIONS

$$
\begin{aligned}
& \Delta_{4}^{g}=-\left(\check{c}_{1}^{22} e^{v_{1}(1-\xi)}+\check{c}_{2}^{22} e^{v_{2}(1-\xi)}+\check{c}_{3}^{22} e^{v_{3}(1-\xi)}+\check{c}_{4}^{22} e^{v_{4}(1-\xi)}\right) \\
& \times \operatorname{det}\left(\begin{array}{ccccc}
e^{\mu_{1}(1-\xi)} & e^{\mu_{2}(1-\xi)} & 0 & 0 & 0 \\
0 & 0 & \left(v_{1}\right)^{2} e^{v_{1}(1-\xi)} & \left(v_{3}\right)^{2} e^{v_{3}(1-\xi)} & \left(v_{4}\right)^{2} e^{v_{4}(1-\xi)} \\
0 & 0 & \left(v_{1}\right)^{2} e^{-v_{1} \xi} & \left(v_{3}\right)^{2} e^{-v_{3} \xi} & \left(v_{4}\right)^{2} e^{-v_{4} \xi} \\
-e^{-\mu_{1} \xi} & -e^{-\mu_{2} \xi} & e^{-v_{1} \xi} & e^{-v_{3} \xi} & e^{-v_{4} \xi} \\
-\mu_{1} e^{-\mu_{1} \xi} & -\mu_{2} e^{-\mu_{2} \xi} & \left(v_{1}\right)^{3} e^{-v_{1} \xi} & \left(v_{3}\right)^{3} e^{-v_{3} \xi} & \left(v_{4}\right)^{3} e^{-v_{4} \xi}
\end{array}\right) \\
& +\left(\left(v_{1}\right)^{2} \breve{c}_{1}^{22} e^{v_{1}(1-\xi)}+\left(v_{2}\right)^{2} \check{c}_{2}^{22} e^{v_{2}(1-\xi)}+\left(v_{3}\right)^{2} \breve{c}_{3}^{22} e^{v_{3}(1-\xi)}+\left(v_{4}\right)^{2} \breve{c}_{4}^{22} e^{v_{4}(1-\xi)}\right) \\
& \times \operatorname{det}\left(\begin{array}{ccccc}
e^{\mu_{1}(1-\xi)} & e^{\mu_{2}(1-\xi)} & 0 & 0 & 0 \\
0 & 0 & e^{v_{1}(1-\xi)} & e^{v_{3}(1-\xi)} & e^{v_{4}(1-\xi)} \\
0 & 0 & \left(v_{1}\right)^{2} e^{-v_{1} \xi} & \left(v_{3}\right)^{2} e^{-v_{3} \xi} & \left(v_{4}\right)^{2} e^{-v_{4} \xi} \\
-e^{-\mu_{1} \xi} & -e^{-\mu_{2} \xi} & e^{-v_{1} \xi} & e^{-v_{3} \xi} & e^{-v_{4} \xi} \\
-\mu_{1} e^{-\mu_{1} \xi} & -\mu_{2} e^{-\mu_{2} \xi} & \left(v_{1}\right)^{3} e^{-v_{1} \xi} & \left(v_{3}\right)^{3} e^{-v_{3} \xi} & \left(v_{4}\right)^{3} e^{-v_{4} \xi}
\end{array}\right) \\
& =-e^{-v_{1} \xi}\left[\check{c}_{1}^{22} e^{v_{1}(1-\xi)}+\check{c}_{2}^{22} e^{v_{2}(1-\xi)}+\check{c}_{3}^{22} e^{v_{3}(1-\xi)}+\check{c}_{4}^{22} e^{v_{4}(1-\xi)}\right] \\
& \times\left[\lambda^{\frac{7}{2}}\left(e^{\mu_{2}}-e^{\mu_{1}}\right)\left((1+\mathrm{i}) e^{\mathrm{i} \sqrt{\lambda}}+(\mathrm{i}-1) e^{-\mathrm{i} \sqrt{\lambda}}-2 \mathrm{i} e^{\sqrt{\lambda}}\right)-2 \lambda^{2}\left(\mu_{1} e^{\mu_{2}}-\mu_{2} e^{\mu_{1}}\right)\left(e^{\mathrm{i} \sqrt{\lambda}}-e^{-\mathrm{i} \sqrt{\lambda}}\right)\right] \\
& +e^{-v_{1} \xi}\left[\left(v_{1}\right)^{2} \breve{c}_{1}^{22} e^{v_{1}(1-\xi)}+\left(v_{2}\right)^{2} \breve{c}_{2}^{22} e^{v_{2}(1-\xi)}+\left(v_{3}\right)^{2} \check{c}_{3}^{22} e^{v_{3}(1-\xi)}+\left(v_{4}\right)^{2} \breve{c}_{4}^{22} e^{v_{4}(1-\xi)}\right] \\
& \times\left[\lambda^{\frac{5}{2}}\left(e^{\mu_{2}}-e^{\mu_{1}}\right)\left((1-\mathrm{i}) e^{-\mathrm{i} \sqrt{\lambda}}-2 \mathrm{i} e^{\sqrt{\lambda}}-(1+\mathrm{i}) e^{\mathrm{i} \sqrt{\lambda}}\right)\right. \\
& \left.-2 \lambda\left(\mu_{1} e^{\mu_{2}}-\mu_{2} e^{\mu_{1}}\right)\left(e^{-\mathrm{i} \sqrt{\lambda}}-e^{\mathrm{i} \sqrt{\lambda}}\right)\right], \\
& \Delta_{5}^{g}=e^{-v_{4} \xi}\left[\check{c}_{1}^{22} e^{v_{1}(1-\xi)}+\check{c}_{2}^{22} e^{v_{2}(1-\xi)}+\check{c}_{3}^{22} e^{v_{3}(1-\xi)}+\check{c}_{4}^{22} e^{v_{4}(1-\xi)}\right] \\
& \times\left[\lambda^{\frac{7}{2}}\left(e^{\mu_{2}}-e^{\mu_{1}}\right)\left(2 e^{-\mathrm{i} \sqrt{\lambda}}+(\mathrm{i}-1) e^{\sqrt{\lambda}}-(1+\mathrm{i}) e^{-\sqrt{\lambda}}\right)-2 \lambda^{2}\left(\mu_{1} e^{\mu_{2}}-\mu_{2} e^{\mu_{1}}\right)\left(e^{\sqrt{\lambda}}-e^{-\sqrt{\lambda}}\right)\right] \\
& -e^{-v_{4} \xi}\left[\left(v_{1}\right)^{2} \check{c}_{1}^{22} e^{v_{1}(1-\xi)}+\left(v_{2}\right)^{2} \check{c}_{2}^{22} e^{v_{2}(1-\xi)}+\left(v_{3}\right)^{2} \check{c}_{3}^{22} e^{v_{3}(1-\xi)}+\left(v_{4}\right)^{2} \check{c}_{4}^{22} e^{v_{4}(1-\xi)}\right] \\
& \times\left[\lambda^{\frac{5}{2}}\left(e^{\mu_{2}}-e^{\mu_{1}}\right)\left((\mathrm{i}-1) e^{-\sqrt{\lambda}}-2 e^{-\mathrm{i} \sqrt{\lambda}}-(1+\mathrm{i}) e^{\sqrt{\lambda}}\right)\right. \\
& \left.-2 \lambda\left(\mu_{1} e^{\mu_{2}}-\mu_{2} e^{\mu_{1}}\right)\left(e^{\sqrt{\lambda}}-e^{-\sqrt{\lambda}}\right)\right],
\end{aligned}
$$

and

$$
\begin{aligned}
\Delta_{6}^{g}= & -e^{-v_{3} \xi}\left[\check{c}_{1}^{22} e^{v_{1}(1-\xi)}+\check{c}_{2}^{22} e^{v_{2}(1-\xi)}+\check{c}_{3}^{22} e^{v_{3}(1-\xi)}+\check{c}_{4}^{22} e^{v_{4}(1-\xi)}\right] \\
& \times\left[\lambda^{\frac{7}{2}}\left(e^{\mu_{2}}-e^{\mu_{1}}\right)\left(2 e^{\mathrm{i} \sqrt{\lambda}}+(\mathrm{i}-1) e^{-\sqrt{\lambda}}-(1+\mathrm{i}) e^{\sqrt{\lambda}}\right)-2 \lambda^{2}\left(\mu_{1} e^{\mu_{2}}-\mu_{2} e^{\mu_{1}}\right)\left(e^{\sqrt{\lambda}}-e^{-\sqrt{\lambda}}\right)\right] \\
& +e^{-v_{3} \xi}\left[\left(v_{1}\right)^{2} \check{c}_{1}^{22} e^{v_{1}(1-\xi)}+\left(v_{2}\right)^{2} \check{c}_{2}^{22} e^{v_{2}(1-\xi)}+\left(v_{3}\right)^{2} \breve{c}_{3}^{22} e^{\nu_{3}(1-\xi)}+\left(v_{4}\right)^{2} \breve{c}_{4}^{22} e^{v_{4}(1-\xi)}\right] \\
& \times\left[\lambda^{\frac{5}{2}}\left(e^{\mu_{2}}-e^{\mu_{1}}\right)\left((\mathrm{i}-1) e^{-\sqrt{\lambda}}-2 e^{-\mathrm{i} \sqrt{\lambda}}-(1+\mathrm{i}) e^{\sqrt{\lambda}}\right)\right. \\
& \left.-2 \lambda\left(\mu_{1} e^{\mu_{2}}-\mu_{2} e^{\mu_{1}}\right)\left(e^{\sqrt{\lambda}}-e^{-\sqrt{\lambda}}\right)\right] .
\end{aligned}
$$

The proof is complete.

Lemma 3.2. There exists a $C>0$ such that

$$
|\Delta| \geqslant C|\lambda|^{\frac{7}{2}} e^{\sqrt{2|\lambda|}}, \quad \forall \lambda \in \mathrm{i} \mathbb{R}
$$


where $\Delta$ is given as in (3.7).

Proof. It is ready to find that, for every $\lambda \in \mathrm{i} \mathbb{R}$,

$$
\begin{aligned}
& \left|4 \lambda^{2}\left(\mu_{1} e^{\mu_{2}}-\mu_{2} e^{\mu_{1}}\right)\left[-e^{(1-\mathrm{i}) \sqrt{\lambda}}+e^{(1+\mathrm{i}) \sqrt{\lambda}}+e^{-(1+\mathrm{i}) \sqrt{\lambda}}-e^{(-1+\mathrm{i}) \sqrt{\lambda}}\right]\right| \\
\leqslant & C|\lambda|^{3}\left[\left|e^{(1-\mathrm{i}) \sqrt{\lambda}}\right|+\left|e^{(1+\mathrm{i}) \sqrt{\lambda}}\right|+\left|e^{-(1+\mathrm{i}) \sqrt{\lambda}}\right|+\left|e^{(-1+\mathrm{i}) \sqrt{\lambda}}\right|\right] \\
= & C|\lambda|^{3}\left(e^{\sqrt{2|\lambda|}}+e^{-\sqrt{2|\lambda|}}+2\right) .
\end{aligned}
$$

Therefore,

$$
\lim _{\lambda \in \mathbb{R},|\lambda| \rightarrow+\infty} \frac{\left|4 \lambda^{2}\left(\mu_{1} e^{\mu_{2}}-\mu_{2} e^{\mu_{1}}\right)\left[-e^{(1-\mathrm{i}) \sqrt{\lambda}}+e^{(1+\mathrm{i}) \sqrt{\lambda}}+e^{-(1+\mathrm{i}) \sqrt{\lambda}}-e^{(-1+\mathrm{i}) \sqrt{\lambda}}\right]\right|}{|\lambda|^{\frac{7}{2}} e^{\sqrt{2|\lambda|}}}=0 .
$$

On the other hand, we have

$$
\begin{aligned}
& \quad\left|2(1+\mathrm{i}) \lambda^{\frac{7}{2}}\left(e^{\mu_{1}}-e^{\mu_{2}}\right)\left[\mathrm{i} e^{(1+\mathrm{i}) \sqrt{\lambda}}-\mathrm{i} e^{-(1+\mathrm{i}) \sqrt{\lambda}}-e^{(-1+\mathrm{i}) \sqrt{\lambda}}\right]\right| \\
& \leqslant C|\lambda|^{\frac{7}{2}}\left(e^{-\sqrt{2|\lambda|}}+2\right) \quad \text { for every } \lambda \in \mathrm{i} \mathbb{R} \text { with } \mathfrak{I m} \lambda>0
\end{aligned}
$$

and

$$
\begin{aligned}
& \quad\left|2(1+\mathrm{i}) \lambda^{\frac{7}{2}}\left(e^{\mu_{1}}-e^{\mu_{2}}\right)\left[e^{(1-\mathrm{i}) \sqrt{\lambda}}-\mathrm{i} e^{-(1+\mathrm{i}) \sqrt{\lambda}}-e^{(-1+\mathrm{i}) \sqrt{\lambda}}\right]\right| \\
& \leqslant C|\lambda|^{\frac{7}{2}}\left(e^{-\sqrt{2|\lambda|}}+2\right) \quad \text { for every } \lambda \in \mathrm{i} \mathbb{R} \text { with } \mathfrak{I m} \lambda<0 .
\end{aligned}
$$

These two observations imply immediately

$$
\lim _{\lambda \in \mathbb{R}, \mathfrak{I m} \lambda \rightarrow+\infty} \frac{2(1+\mathrm{i}) \lambda^{\frac{7}{2}}\left(e^{\mu_{1}}-e^{\mu_{2}}\right)\left[\mathrm{i} e^{(1+\mathrm{i}) \sqrt{\lambda}}-\mathrm{i} e^{-(1+\mathrm{i}) \sqrt{\lambda}}-e^{(-1+\mathrm{i}) \sqrt{\lambda}}\right]}{|\lambda|^{\frac{7}{2}} e^{\sqrt{2|\lambda|}}}=0,
$$

and

$$
\lim _{\lambda \in \mathbb{R}, \mathfrak{I m} \lambda \rightarrow-\infty} \frac{2(1+\mathrm{i}) \lambda^{\frac{7}{2}}\left(e^{\mu_{1}}-e^{\mu_{2}}\right)\left[e^{(1-\mathrm{i}) \sqrt{\lambda}}-\mathrm{i} e^{-(1+\mathrm{i}) \sqrt{\lambda}}-e^{(-1+\mathrm{i}) \sqrt{\lambda}}\right]}{|\lambda|^{\frac{7}{2}} e^{\sqrt{2|\lambda|}}}=0,
$$

respectively. By using (3.3), we have

$$
\begin{aligned}
\lim _{\lambda \in \mathbb{R},|\lambda| \rightarrow+\infty}\left|e^{2 \mu_{2}}\right| & =\lim _{\lambda \in \mathbb{R},|\lambda| \rightarrow+\infty}\left|\exp \left(-\lambda-\frac{a}{2}+\frac{a^{2}}{8 \lambda}+\mathscr{O}\left(|\lambda|^{-2}\right)\right)\right| \\
& =\lim _{\lambda \in \mathbb{R} R,|\lambda| \rightarrow+\infty} \exp \left(-\frac{a}{2}+\Re \mathfrak{e} \mathscr{O}\left(|\lambda|^{-2}\right)\right)=e^{-\frac{a}{2}} .
\end{aligned}
$$

With the aid of this, and (3.20), (3.21), and (3.22), we can obtain the desired estimate

$$
\begin{aligned}
|\Delta|= & \mid 2(1+\mathrm{i}) \lambda^{\frac{7}{2}}\left(e^{\mu_{1}}-e^{\mu_{2}}\right)\left[e^{(1-\mathrm{i}) \sqrt{\lambda}}+\mathrm{i} e^{(1+\mathrm{i}) \sqrt{\lambda}}-\mathrm{i} e^{-(1+\mathrm{i}) \sqrt{\lambda}}-e^{(-1+\mathrm{i}) \sqrt{\lambda}}\right] \\
& +4 \lambda^{2}\left(\mu_{1} e^{\mu_{2}}-\mu_{2} e^{\mu_{1}}\right)\left[-e^{(1-\mathrm{i}) \sqrt{\lambda}}+e^{(1+\mathrm{i}) \sqrt{\lambda}}+e^{-(1+\mathrm{i}) \sqrt{\lambda}}-e^{(-1+\mathrm{i}) \sqrt{\lambda}}\right] \mid \\
\geqslant & \left|2(1+\mathrm{i}) \lambda^{\frac{7}{2}}\left(e^{\mu_{1}}-e^{\mu_{2}}\right)\left[e^{(1-\mathrm{i}) \sqrt{\lambda}}+\mathrm{i} e^{(1+\mathrm{i}) \sqrt{\lambda}}-\mathrm{i} e^{-(1+\mathrm{i}) \sqrt{\lambda}}-e^{(-1+\mathrm{i}) \sqrt{\lambda}}\right]\right| \\
& -\left|4 \lambda^{2}\left(\mu_{1} e^{\mu_{2}}-\mu_{2} e^{\mu_{1}}\right)\left[-e^{(1-\mathrm{i}) \sqrt{\lambda}}+e^{(1+\mathrm{i}) \sqrt{\lambda}}+e^{-(1+\mathrm{i}) \sqrt{\lambda}}-e^{(-1+\mathrm{i}) \sqrt{\lambda}}\right]\right| \\
\geqslant & C|\lambda|^{\frac{7}{2}} e^{\sqrt{2|\lambda|}} \text { for } \lambda \in \mathrm{i} \mathbb{R} \text { with }|\lambda| \text { sufficiently large. }
\end{aligned}
$$


Lemma 3.3 (Young's inequality). For every $f \in L^{1}(\mathbb{R})$ and $g \in L^{p}(\mathbb{R})(1 \leqslant p \leqslant+\infty)$, we have $\| f *$ $g\left\|_{L^{p}(\mathbb{R})} \leqslant\right\| f\left\|_{L^{1}(\mathbb{R})}\right\| g \|_{L^{p}(\mathbb{R})}$, in which, $(f * g)(x)=\int_{-\infty}^{+\infty} f(x-y) g(y) d y, \forall x \in \mathbb{R}$.

Proposition 3.2. There exists a $C>0$ such that $\left\|\left(\lambda \operatorname{id}_{\mathscr{H}}-A\right)^{-1}\right\|_{\mathscr{L}(\mathscr{H})} \leqslant C e^{C|\lambda|}, \forall \lambda \in \mathrm{i} \mathbb{R}$, where $\mathrm{id}_{\mathscr{H}}$ is the identity operator on $\mathscr{H}$.

Proof. Let $\lambda \in \mathrm{i} \mathbb{R},(\varphi, \widetilde{\varphi}, \psi, \widetilde{\psi}) \in \mathscr{D}(A)$, and write $(f, \widetilde{f}, g, \widetilde{g})=\left(\lambda \operatorname{id}_{\mathscr{H}}-A\right)(\varphi, \widetilde{\varphi}, \psi, \widetilde{\psi}) .(\varphi, \widetilde{\varphi}, \psi, \widetilde{\psi}) \in$ $\mathscr{D}(A)$ and $(f, \widetilde{f}, g, \widetilde{g})$ are such that

$$
\begin{array}{ll}
\lambda \varphi-\widetilde{\varphi}=f & \text { in }(0,1), \\
\lambda \widetilde{\varphi}-\varphi^{\prime \prime}+a \widetilde{\varphi}=\widetilde{f} & \text { in }(0,1), \\
\lambda \psi-\widetilde{\psi}=g & \text { in }(0,1), \\
\lambda \widetilde{\psi}+\psi^{(4)}=\widetilde{g} & \text { in }(0,1), \\
\varphi(0)-\psi(0)=\varphi^{\prime}(0)-\psi^{\prime \prime \prime}(0)=0, & \\
\varphi(1)=\psi(1)=\psi^{\prime \prime}(0)=\psi^{\prime \prime}(1)=0, &
\end{array}
$$

or equivalently

$$
\left.\begin{array}{ll}
\widetilde{\varphi}=\lambda \varphi-f & \text { in }(0,1), \\
\varphi^{\prime \prime}-\left(\lambda^{2}+a \lambda\right) \varphi=-(\lambda+a) f-\widetilde{f} & \text { in }(0,1), \\
\widetilde{\psi}=\lambda \psi-g & \text { in }(0,1), \\
\psi^{(4)}+\lambda^{2} \psi=\lambda g+\widetilde{g} & \text { in }(0,1), \\
\varphi(0)-\psi(0)=\varphi^{\prime}(0)-\psi^{\prime \prime \prime}(0)=0, & \\
\varphi(1)=\psi(1)=\psi^{\prime \prime}(0)=\psi^{\prime \prime}(1)=0 . &
\end{array}\right\}
$$

By Lemma 3.1, $\varphi$ and $\psi$ can be represented respectively by

$$
\begin{aligned}
& \varphi(x)=\int_{0}^{1} G^{11}(x, \xi)(-(\lambda+a) f-\widetilde{f})(\xi) d \xi+\int_{0}^{1} G^{12}(x, \xi)(\lambda g+\widetilde{g})(\xi) d \xi \text { and } \\
& \psi(x)=\int_{0}^{1} G^{21}(x, \xi)(-(\lambda+a) f-\widetilde{f})(\xi) d \xi+\int_{0}^{1} G^{22}(x, \xi)(\lambda g+\widetilde{g})(\xi) d \xi
\end{aligned}
$$

To accomplish the proof of Proposition 3.2, it is essential to obtain the estimate $\|(\varphi, 0, \psi, 0)\|_{\mathscr{H}} \leqslant$ $C\|(f, \widetilde{f}, g, \widetilde{g})\|_{\mathscr{H}}$ and to find an upper bound on the constant $C$. Let us start this long procedure by analyzing the term $\int_{0}^{1} G^{11}(x, \xi)(-(\lambda+a) f-\widetilde{f})(\xi) d \xi$. We have by some routine calculations that

$$
\begin{aligned}
& \left\|\int_{0}^{x} e^{\mu_{2}(1-2 \xi)} e^{\mu_{1}(x-\xi)}(-(\lambda+a) f-\widetilde{f})(\xi) d \xi\right\|_{L^{2}(0,1)} \\
& =\left\|e^{\mu_{2}(1-2 x)} \int_{0}^{x} e^{-\left(\mu_{1}+\mu_{2}\right) \xi} e^{\mu_{2}(x-\xi)}(-(\lambda+a) f-\widetilde{f})(\xi) d \xi\right\|_{L^{2}(0,1)} \\
& \leqslant C\left\|\int_{0}^{x} e^{\mu_{2}(x-\xi)}(-(\lambda+a) f-\widetilde{f})(\xi) d \xi\right\|_{L^{2}(0,1)} \\
& \leqslant C\left\|e^{\mu_{2} x}\right\|_{L_{x}^{1}(0,1)}\|-(\lambda+a) f-\widetilde{f}\|_{L^{2}(0,1)} \\
& \leqslant C\left[(|\lambda|+a)\|f\|_{L^{2}(0,1)}+\|\widetilde{f}\|_{L^{2}(0,1)}\right]
\end{aligned}
$$


where the " $\leqslant$ " in the third line follows from the fact that $\Re \mathfrak{e} \lambda$ is bounded and from the algebraic identity $\mu_{1}+\mu_{2}=0$, while the " $\leqslant$ " in the forth line follows from Young's inequality. With the aid of (3.4), (3.5), (3.6), (3.8), (3.9), (3.27) and Lemma 3.2, we have by some routine calculations that

$$
\begin{aligned}
& \left\|\int_{0}^{x}\left(c_{1}^{11}+\check{c}_{1}^{11}\right) e^{\mu_{1}(x-\xi)}(-(\lambda+a) f-\widetilde{f})(\xi) d \xi\right\|_{L^{2}(0,1)} \\
& \leqslant\left\|\int_{0}^{x} c_{1}^{11} e^{\mu_{1}(x-\xi)}(-(\lambda+a) f-\widetilde{f})(\xi) d \xi\right\|_{L^{2}(0,1)} \\
& +\left\|\int_{0}^{x} \check{c}_{1}^{11} e^{\mu_{1}(x-\xi)}(-(\lambda+a) f-\widetilde{f})(\xi) d \xi\right\|_{L^{2}(0,1)} \\
& \leqslant \frac{\left|4 \lambda^{2} \mu_{2} \check{c}_{1}^{11} e^{\mu_{1}}\left[-e^{(1-\mathrm{i}) \sqrt{\lambda}}+e^{(1+\mathrm{i}) \sqrt{\lambda}}+e^{-(1+\mathrm{i}) \sqrt{\lambda}}-e^{(-1+\mathrm{i}) \sqrt{\lambda}}\right]\right|}{|\Delta|} \\
& \times\left\|\int_{0}^{x} e^{\mu_{1}(x-\xi)}(-(\lambda+a) f-\widetilde{f})(\xi) d \xi\right\|_{L^{2}(0,1)} \\
& +\frac{\mid 4 \lambda^{2} \mu_{2} \check{c}_{2}^{11}\left[-e^{(1-\mathrm{i}) \sqrt{\lambda}}+e^{(1+\mathrm{i}) \sqrt{\lambda}}+e^{-(1+\mathrm{i}) \sqrt{\lambda}}-e^{(-1+\mathrm{i}) \sqrt{\lambda}]}\right]}{|\Delta|} \\
& \times\left\|\int_{0}^{x} e^{\mu_{2}(1-2 \xi)} e^{\mu_{1}(x-\xi)}(-(\lambda+a) f-\widetilde{f})(\xi) d \xi\right\|_{L^{2}(0,1)} \\
& +\frac{\left|-2 \lambda \frac{7}{2}(1+\mathrm{i}) \check{c}_{1}^{11} e^{\mu_{1}}\left[e^{(1-\mathrm{i}) \sqrt{\lambda}}+\mathrm{i} e^{(1+\mathrm{i}) \sqrt{\lambda}}-\mathrm{i} e^{-(1+\mathrm{i}) \sqrt{\lambda}}-e^{(-1+\mathrm{i}) \sqrt{\lambda}}\right]\right|}{|\Delta|} \\
& \times\left\|\int_{0}^{x} e^{\mu_{1}(x-\xi)}(-(\lambda+a) f-\tilde{f})(\xi) d \xi\right\|_{L^{2}(0,1)} \\
& +\frac{\left|-2 \lambda^{\frac{7}{2}}(1+\mathrm{i}) \check{c}_{2}^{11}\left[e^{(1-\mathrm{i}) \sqrt{\lambda}}+\mathrm{i} e^{(1+\mathrm{i}) \sqrt{\lambda}}-\mathrm{i} e^{-(1+\mathrm{i}) \sqrt{\lambda}}-e^{(-1+\mathrm{i}) \sqrt{\lambda}}\right]\right|}{|\Delta|} \\
& \times\left\|\int_{0}^{x} e^{\mu_{2}(1-2 \xi)} e^{\mu_{1}(x-\xi)}(-(\lambda+a) f-\widetilde{f})(\xi) d \xi\right\|_{L^{2}(0,1)} \\
& +\frac{C}{|\lambda|}\left[(|\lambda|+a)\|f\|_{L^{2}(0,1)}+\|\widetilde{f}\|_{L^{2}(0,1)}\right] \\
& \leqslant C\left(|\lambda|^{-1}+|\lambda|^{-\frac{3}{4}}\right)\left[(|\lambda|+a)\|f\|_{L^{2}(0,1)}+\|\widetilde{f}\|_{L^{2}(0,1)}\right] \\
& \leqslant C\left(\|f\|_{L^{2}(0,1)}+\|\widetilde{f}\|_{L^{2}(0,1)}\right) \text {, }
\end{aligned}
$$

where the " $\leqslant$ " in the penultimate line follows from Lemma 3.2. Similarly, we have

$$
\left\|\int_{0}^{x}\left(c_{2}^{11}+\check{c}_{2}^{11}\right) e^{\mu_{2}(x-\xi)}(-(\lambda+a) f-\widetilde{f})(\xi) d \xi\right\|_{L^{2}(0,1)} \leqslant C\left(\|f\|_{L^{2}(0,1)}+\|\widetilde{f}\|_{L^{2}(0,1)}\right) .
$$

Combine (3.28) and (3.29), we obtain

$$
\left\|\int_{0}^{x} G^{11}(x, \xi)(-(\lambda+a) f-\widetilde{f})(\xi) d \xi\right\|_{L^{2}(0,1)} \leqslant C\left(\|f\|_{L^{2}(0,1)}+\|\widetilde{f}\|_{L^{2}(0,1)}\right) .
$$


Now it turns to analyze $\int_{x}^{1} G^{11}(x, \xi)(-(\lambda+a) f-\widetilde{f})(\xi) d \xi$. By changing of variables, we have

$$
\begin{aligned}
& \left\|\int_{x}^{1} e^{\mu_{1}(x-\xi)}(-(\lambda+a) f-\widetilde{f})(\xi) d \xi\right\|_{L^{2}(0,1)} \\
= & \left\|\int_{0}^{1-x} e^{-\mu_{1}((1-x)-\xi)}(-(\lambda+a) f-\widetilde{f})(1-\xi) d \xi\right\|_{L^{2}(0,1)} \\
= & \left\|\int_{0}^{x} e^{-\mu_{1}(x-\xi)}(-(\lambda+a) f-\widetilde{f})(1-\xi) d \xi\right\|_{L^{2}(0,1)} \\
\leqslant & \left\|e^{-\mu_{1} x}\right\|_{L_{x}^{1}(0,1)}\|-(\lambda+a) f-\widetilde{f}\|_{L^{2}(0,1)} \\
\leqslant & C\left[(|\lambda|+a)\|f\|_{L^{2}(0,1)}+\|\widetilde{f}\|_{L^{2}(0,1)}\right] .
\end{aligned}
$$

It follows that

$$
\begin{aligned}
& \left\|\int_{x}^{1} e^{\mu_{2}(1-2 \xi)} e^{\mu_{1}(x-\xi)}(-(\lambda+a) f-\widetilde{f})(\xi) d \xi\right\|_{L^{2}(0,1)} \\
& =\left\|e^{\mu_{2}(1-2 x)} \int_{x}^{1} e^{\mu_{2}(x-\xi)}(-(\lambda+a) f-\widetilde{f})(\xi) d \xi\right\|_{L^{2}(0,1)} \\
& \leqslant C\left\|e^{-\mu_{2} x}\right\|_{L_{x}^{1}(0,1)}\|-(\lambda+a) f-\widetilde{f}\|_{L^{2}(0,1)} \\
& \leqslant C\left[(|\lambda|+a)\|f\|_{L^{2}(0,1)}+\|\widetilde{f}\|_{L^{2}(0,1)}\right] .
\end{aligned}
$$

Taking the same route as in (3.28), we have

$$
\begin{aligned}
& \left\|\int_{x}^{1} c_{1}^{11} e^{\mu_{1}(x-\xi)}(-(\lambda+a) f-\widetilde{f})(\xi) d \xi\right\|_{L^{2}(0,1)} \\
\leqslant & \frac{\left|4 \lambda^{2} \mu_{2} \check{c}_{1}^{11} e^{\mu_{1}}\left[-e^{(1-\mathrm{i}) \sqrt{\lambda}}+e^{(1+\mathrm{i}) \sqrt{\lambda}}+e^{-(1+\mathrm{i}) \sqrt{\lambda}}-e^{(-1+\mathrm{i}) \sqrt{\lambda}}\right]\right|}{|\Delta|} \\
& \times\left\|\int_{x}^{1} e^{\mu_{1}(x-\xi)}(-(\lambda+a) f-\widetilde{f})(\xi) d \xi\right\|_{L^{2}(0,1)} \\
& +\frac{\mid 4 \lambda^{2} \mu_{2} \check{c}_{2}^{11}\left[-e^{(1-\mathrm{i}) \sqrt{\lambda}}+e^{(1+\mathrm{i}) \sqrt{\lambda}}+e^{-(1+\mathrm{i}) \sqrt{\lambda}}-e^{(-1+\mathrm{i}) \sqrt{\lambda}] \mid}\right.}{|\Delta|} \\
& \times\left\|\int_{x}^{1} e^{\mu_{2}(1-2 \xi)} e^{\mu_{1}(x-\xi)}(-(\lambda+a) f-\widetilde{f})(\xi) d \xi\right\|_{L^{2}(0,1)} \\
\leqslant & C\left(|\lambda|^{-1}+|\lambda|^{-\frac{3}{4}}\right)\left[(|\lambda|+a)\|f\|_{L^{2}(0,1)}+\|\widetilde{f}\|_{L^{2}(0,1)}\right] \\
\leqslant & C\left(\|f\|_{L^{2}(0,1)}+\|\widetilde{f}\|_{L^{2}(0,1)} .\right.
\end{aligned}
$$

We also have

$$
\left\|\int_{x}^{1} c_{2}^{11} e^{\mu_{2}(x-\xi)}(-(\lambda+a) f-\widetilde{f})(\xi) d \xi\right\|_{L^{2}(0,1)} \leqslant C\left(\|f\|_{L^{2}(0,1)}+\|\widetilde{f}\|_{L^{2}(0,1)}\right) .
$$

This, together with (3.32), implies

$$
\left\|\int_{x}^{1} G^{11}(x, \xi)(-(\lambda+a) f-\widetilde{f})(\xi) d \xi\right\|_{L^{2}(0,1)} \leqslant C\left(\|f\|_{L^{2}(0,1)}+\|\widetilde{f}\|_{L^{2}(0,1)}\right),
$$


which, together with (3.30), implies

$$
\left\|\int_{0}^{1} G^{11}(x, \xi)(-(\lambda+a) f-\widetilde{f})(\xi) d \xi\right\|_{L^{2}(0,1)} \leqslant C\left(\|f\|_{L^{2}(0,1)}+\|\widetilde{f}\|_{L^{2}(0,1)}\right) .
$$

By mimicking the steps in obtaining (3.33), we can prove

$$
\left\|\int_{0}^{1} \partial_{x} G^{11}(x, \xi)(-(\lambda+a) f-\widetilde{f})(\xi) d \xi\right\|_{L^{2}(0,1)} \leqslant C|\lambda|\left(\|f\|_{L^{2}(0,1)}+\|\widetilde{f}\|_{L^{2}(0,1)}\right) .
$$

Let us now analyze $\int_{0}^{1} G^{12}(x, \xi)(\lambda g+\widetilde{g})(\xi) d \xi$. Using (3.4), (3.5), (3.6), (3.14), (3.15), and Lemma 3.2, we have

$$
\begin{aligned}
& \left\|\int_{0}^{1} c_{1}^{12} e^{\mu_{1}(x-\xi)}(\lambda g+\widetilde{g})(\xi) d \xi\right\|_{L^{2}(0,1)} \\
\leqslant & \left\|e^{\mu_{1} x}\right\|_{L_{x}^{2}(0,1)} \mid \frac{4 \lambda \frac{7}{2} e^{\mu_{2}}\left(e^{v_{4}}+\mathrm{i} e^{v_{1}}-e^{v_{3}}-\mathrm{i} e^{v_{2}}\right)}{\Delta} \\
& \times \int_{0}^{1}\left[\check{c}_{1}^{22} e^{v_{1}(1-\xi)}+\check{c}_{2}^{22} e^{v_{2}(1-\xi)}+\check{c}_{3}^{22} e^{v_{3}(1-\xi)}+\check{c}_{4}^{22} e^{v_{4}(1-\xi)}\right](\lambda g+\widetilde{g})(\xi) d \xi \mid \\
& +\left\|e^{\mu_{1} x}\right\|_{L_{x}^{2}(0,1)} \mid \frac{4 \lambda \frac{5}{2} e^{\mu_{2}}\left(e^{v_{4}}+\mathrm{i}^{v_{1}}-e^{v_{3}}-\mathrm{i} e^{v_{2}}\right)}{\Delta} \\
& \times \int_{0}^{1}\left[\left(v_{1}\right)^{2} \check{c}_{1}^{22} e^{v_{1}(1-\xi)}+\left(v_{2}\right)^{2} \check{c}_{2}^{22} e^{v_{2}(1-\xi)}+\left(v_{3}\right)^{2} \check{c}_{3}^{22} e^{v_{3}(1-\xi)}+\left(v_{4}\right)^{2} \check{c}_{4}^{22} e^{v_{4}(1-\xi)}\right](\lambda g+\widetilde{g})(\xi) d \xi \\
\leqslant & C \frac{|\lambda|^{2}\left(e^{\frac{\sqrt{2|\lambda|}}{2}}\right)^{2}}{|\Delta|}\|\lambda g+\widetilde{g}\|_{L^{2}(0,1)} \\
\leqslant & \frac{C}{\sqrt{|\lambda|}}\left(\|g\|_{L^{2}(0,1)}+\|\widetilde{g}\|_{L^{2}(0,1)}\right) .
\end{aligned}
$$

With similar routine calculations, we also have

$$
\left\|\int_{0}^{1} c_{2}^{12} e^{\mu_{2}(x-\xi)}(\lambda g+\widetilde{g})(\xi) d \xi\right\|_{L^{2}(0,1)} \leqslant \frac{C}{\sqrt{|\lambda|}}\left(\|g\|_{L^{2}(0,1)}+\|\widetilde{g}\|_{L^{2}(0,1)}\right) .
$$

This, along with 3.35 , implies

$$
\left\|\int_{0}^{1} G^{12}(x, \xi)(\lambda g+\widetilde{g})(\xi) d \xi\right\|_{L^{2}(0,1)} \leqslant \frac{C}{\sqrt{|\lambda|}}\left(\|g\|_{L^{2}(0,1)}+\|\widetilde{g}\|_{L^{2}(0,1)}\right) .
$$

By mimicking steps as in deriving estimate (3.36), we have

$$
\left\|\int_{0}^{1} \partial_{x} G^{12}(x, \xi)(\lambda g+\widetilde{g})(\xi) d \xi\right\|_{L^{2}(0,1)} \leqslant C \sqrt{|\lambda|}\left(\|g\|_{L^{2}(0,1)}+\|\widetilde{g}\|_{L^{2}(0,1)}\right) .
$$


In view of (3.4), (3.5), (3.6), (3.10), (3.11), (3.12), (3.13), and Lemma 3.2, we have

$$
\begin{aligned}
& \left\|\int_{0}^{1} c_{1}^{21} e^{v_{1}(x-\xi)}(-(\lambda+a) f-\widetilde{f})(\xi) d \xi\right\|_{L^{2}(0,1)} \\
\leqslant & \left\|e^{v_{1} x}\right\|_{L^{2}(0,1)} \mid \frac{2 \lambda^{2}\left(\mu_{1}-\mu_{2}\right)\left[e^{(-1+\mathrm{i}) \sqrt{\lambda}}-e^{-(1+\mathrm{i}) \sqrt{\lambda}]}\right.}{\Delta} \\
& \times \int_{0}^{1}\left(\check{c}_{1}^{11} e^{\mu_{1}(1-\xi)}+\check{c}_{2}^{11} e^{\mu_{2}(1-\xi)}\right)(-(\lambda+a) f-\widetilde{f})(\xi) d \xi \mid \\
\leqslant & \frac{C}{\sqrt{|\lambda|}} e^{\frac{\sqrt{2|\lambda|}}{2}}\left(\|f\|_{L^{2}(0,1)}+\|\widetilde{f}\|_{L^{2}(0,1)}\right) .
\end{aligned}
$$

It follows that

$$
\left\|\int_{0}^{1} c_{k}^{21} e^{v_{k}(x-\xi)}(-(\lambda+a) f-\widetilde{f})(\xi) d \xi\right\|_{L^{2}(0,1)} \leqslant \frac{C}{\sqrt{|\lambda|}} e^{\frac{\sqrt{2|\lambda|}}{2}}\left(\|f\|_{L^{2}(0,1)}+\|\widetilde{f}\|_{L^{2}(0,1)}\right), \quad k=2,3,4 .
$$

This, together with (3.38), implies

$$
\left\|\int_{0}^{1} G^{21}(\cdot, \xi) f(\xi) d \xi\right\|_{L^{2}(0,1)} \leqslant \frac{C}{\sqrt{|\lambda|}} e^{\frac{\sqrt{2|\lambda|}}{2}}\left(\|f\|_{L^{2}(0,1)}+\|\widetilde{f}\|_{L^{2}(0,1)}\right) .
$$

We also have

$$
\left\|\int_{0}^{1} \partial_{x}^{2} G^{21}(\cdot, \xi) f(\xi) d \xi\right\|_{L^{2}(0,1)} \leqslant C \sqrt{|\lambda|} e^{\frac{\sqrt{2|\lambda|}}{2}}\left(\|f\|_{L^{2}(0,1)}+\|\widetilde{f}\|_{L^{2}(0,1)}\right) .
$$

Let us analyze here $\int_{0}^{1} G^{22}(x, \xi)(\lambda g+\widetilde{g})(\xi) d \xi$. By (3.4), (3.5), (3.6), (3.16), (3.17), (3.18), (3.19), and Lemma 3.2, we have

$$
\begin{aligned}
& \left\|\int_{0}^{x}\left(c_{1}^{22}+\breve{c}_{1}^{22}\right) e^{v_{1}(x-\xi)}(\lambda g+\widetilde{g})(\xi) d \xi\right\|_{L^{2}(0,1)} \\
\leqslant & \frac{C}{\sqrt{|\lambda|}} e^{\frac{\sqrt{2|\lambda|}}{2}}\left(\|g\|_{L^{2}(0,1)}+\|\widetilde{g}\|_{L^{2}(0,1)}\right) \\
& +\left\|e^{v_{1} x}\right\|_{L^{\infty}(0,1)}\left\|\int_{0}^{x} c_{1}^{22} e^{-v_{1} \xi}(\lambda g+\widetilde{g})(\xi) d \xi\right\|_{L^{2}(0,1)} \\
\leqslant & C e^{\frac{\sqrt{2|\lambda|}}{2}}\left[\frac{1}{\sqrt{|\lambda|}}\left(\|g\|_{L^{2}(0,1)}+\|\widetilde{g}\|_{L^{2}(0,1)}\right)+\int_{0}^{1}\left|c_{1}^{22} e^{-v_{1} \xi}(\lambda g+\widetilde{g})(\xi)\right| d \xi\right] \\
\leqslant & C e^{\frac{\sqrt{2|\lambda|}}{2}}\left[\frac{1}{\sqrt{|\lambda|}}\left(\|g\|_{L^{2}(0,1)}+\|\widetilde{g}\|_{L^{2}(0,1)}\right)+\left\|c_{1}^{22} e^{-v_{1} \xi}\right\|_{L_{\xi}^{\infty}(0,1)}\|\lambda g+\widetilde{g}\|_{L^{2}(0,1)}\right] \\
\leqslant & \frac{C}{\sqrt{|\lambda|}} e^{\frac{\sqrt{2|\lambda|}}{2}}\left(\|g\|_{L^{2}(0,1)}+\|\widetilde{g}\|_{L^{2}(0,1)}\right) \\
& +C e^{\frac{\sqrt{2|\lambda|}}{2}}\|\lambda g+\widetilde{g}\|_{L^{2}(0,1)}\left[\frac{|\lambda|^{-\frac{3}{2}} e^{\sqrt{2|\lambda|}}\left(|\lambda|^{\frac{7}{2}}+|\lambda|^{3}\right)}{|\Delta|}+\frac{|\lambda||\lambda|^{-\frac{3}{2}} e^{\sqrt{2|\lambda|}}\left(|\lambda|^{\frac{5}{2}}+|\lambda|^{2}\right)}{|\Delta|}\right] \\
\leqslant & \frac{C}{\sqrt{|\lambda|}} e^{\frac{\sqrt{2|\lambda|}}{2}}\left(\|g\|_{L^{2}(0,1)}+\|\widetilde{g}\|_{L^{2}(0,1)}\right),
\end{aligned}
$$


and

$$
\begin{gathered}
\left\|\int_{0}^{x}\left(c_{k}^{22}+\check{c}_{k}^{22}\right) e^{v_{k}(x-\xi)}(\lambda g+\widetilde{g})(\xi) d \xi\right\|_{L^{2}(0,1)} \\
\leqslant \frac{C}{\sqrt{|\lambda|}} e^{\frac{\sqrt{2|\lambda|}}{2}}\left(\|g\|_{L^{2}(0,1)}+\|\widetilde{g}\|_{L^{2}(0,1)}\right), \quad k=2,3,4 .
\end{gathered}
$$

This, together with (3.41), implies

$$
\left\|\int_{0}^{x} G^{22}(x, \xi)(\lambda g+\widetilde{g})(\xi) d \xi\right\|_{L^{2}(0,1)} \leqslant \frac{C}{\sqrt{|\lambda|}} e^{\frac{\sqrt{2|\lambda|}}{2}}\left(\|g\|_{L^{2}(0,1)}+\|\widetilde{g}\|_{L^{2}(0,1)}\right) .
$$

By applying similar argument as in obtaining (3.41) and (3.42), we can prove

$$
\begin{aligned}
& \left\|\int_{x}^{1} c_{1}^{22} e^{v_{1}(x-\xi)}(\lambda g+\widetilde{g})(\xi) d \xi\right\|_{L^{2}(0,1)} \\
\leqslant & \left\|e^{v_{1} x}\right\|_{L^{\infty}(0,1)}\left\|\int_{x}^{1} c_{1}^{22} e^{-v_{1} \xi}(\lambda g+\widetilde{g})(\xi) d \xi\right\|_{L^{2}(0,1)} \\
\leqslant & C e^{\frac{\sqrt{2|\lambda|}}{2}}\left\|\int_{x}^{1} c_{1}^{22} e^{-v_{1} \xi}(\lambda g+\widetilde{g})(\xi) d \xi\right\|_{L^{\infty}(0,1)} \\
\leqslant & C e^{\frac{\sqrt{2|\lambda|}}{2}} \int_{0}^{1}\left|c_{1}^{22} e^{-v_{1} \xi}(\lambda g+\widetilde{g})(\xi)\right| d \xi \\
\leqslant & C e^{\frac{\sqrt{2|\lambda|}}{2}}\left\|c_{1}^{22} e^{-v_{1} \xi}\right\|_{L_{\xi}^{\infty}(0,1)}\|\lambda g+\widetilde{g}\|_{L^{2}(0,1)} \\
\leqslant & C e^{\frac{\sqrt{2|\lambda|}}{2}}\|\lambda g+\widetilde{g}\|_{L^{2}(0,1)}\left[\frac{|\lambda|^{-\frac{3}{2}} e^{\sqrt{2|\lambda|}}\left(|\lambda|^{\frac{7}{2}}+|\lambda|^{3}\right)}{|\Delta|}+\frac{|\lambda||\lambda|^{-\frac{3}{2}} e^{\sqrt{2|\lambda|}\left(|\lambda|^{\frac{5}{2}}+|\lambda|^{2}\right)}}{|\Delta|}\right. \\
\leqslant & \frac{C}{\sqrt{|\lambda|}} e^{\frac{\sqrt{2|\lambda|}}{2}}\left(\|g\|_{L^{2}(0,1)}+\|\widetilde{g}\|_{L^{2}(0,1)}\right)
\end{aligned}
$$

and

$$
\begin{aligned}
& \left\|\int_{x}^{1} c_{k}^{22} e^{v_{k}(x-\xi)}(\lambda g+\widetilde{g})(\xi) d \xi\right\|_{L^{2}(0,1)} \\
\leqslant & \frac{C}{\sqrt{|\lambda|}} e^{\frac{\sqrt{2|\lambda|}}{2}}\left(\|g\|_{L^{2}(0,1)}+\|\widetilde{g}\|_{L^{2}(0,1)}\right), \quad k=2,3,4,
\end{aligned}
$$

respectively. This, together with 3.44 , implies

$$
\left\|\int_{x}^{1} G^{22}(x, \xi)(\lambda g+\widetilde{g})(\xi) d \xi\right\|_{L^{2}(0,1)} \leqslant \frac{C}{\sqrt{|\lambda|}} e^{\frac{\sqrt{2|\lambda|}}{2}}\left(\|g\|_{L^{2}(0,1)}+\|\widetilde{g}\|_{L^{2}(0,1)}\right),
$$

which, together with (3.43), implies

$$
\left\|\int_{0}^{1} G^{22}(x, \xi)(\lambda g+\widetilde{g})(\xi) d \xi\right\|_{L^{2}(0,1)} \leqslant \frac{C}{\sqrt{|\lambda|}} e^{\frac{\sqrt{2|\lambda|}}{2}}\left(\|g\|_{L^{2}(0,1)}+\|\widetilde{g}\|_{L^{2}(0,1)}\right) .
$$

In a similar way, we can prove

$$
\left\|\int_{0}^{1} \partial_{x}^{2} G^{22}(x, \xi)(\lambda g+\widetilde{g})(\xi) d \xi\right\|_{L^{2}(0,1)} \leqslant C \sqrt{|\lambda|} e^{\frac{\sqrt{2|\lambda|}}{2}}\left(\|g\|_{L^{2}(0,1)}+\|\widetilde{g}\|_{L^{2}(0,1)}\right) .
$$

Combine (3.25), (3.33) and (3.36), we obtain

$$
\|\varphi\|_{L^{2}(0,1)} \leqslant C\left(\|f\|_{L^{2}(0,1)}+\|\widetilde{f}\|_{L^{2}(0,1)}\right)
$$


which, together with $(3.24)_{1}$, implies

$$
\|\widetilde{\varphi}\|_{L^{2}(0,1)} \leqslant C|\lambda|\left(\|f\|_{L^{2}(0,1)}+\|\widetilde{f}\|_{L^{2}(0,1)}\right) .
$$

Combine (3.26), (3.39) and (3.45), to get

$$
\|\psi\|_{L^{2}(0,1)} \leqslant \frac{C}{\sqrt{|\lambda|}} e^{\frac{\sqrt{2|\lambda|}}{2}}\left(\|g\|_{L^{2}(0,1)}+\|\widetilde{g}\|_{L^{2}(0,1)}\right)
$$

which, together with $(3.24)_{3}$, implies

$$
\|\widetilde{\psi}\|_{L^{2}(0,1)} \leqslant C \sqrt{|\lambda|} e^{\frac{\sqrt{2|\lambda|}}{2}}\left(\|g\|_{L^{2}(0,1)}+\|\widetilde{g}\|_{L^{2}(0,1)}\right) .
$$

Combine (3.25), (3.34) and (3.37), we obtain

$$
\left\|\partial_{x} \varphi\right\|_{L^{2}(0,1)} \leqslant C|\lambda|\left(\|f\|_{L^{2}(0,1)}+\|\widetilde{f}\|_{L^{2}(0,1)}\right) .
$$

Combine (3.26), (3.40) and (3.46), we get

$$
\left\|\partial_{x}^{2} \psi\right\|_{L^{2}(0,1)} \leqslant C \sqrt{|\lambda|} e^{\frac{\sqrt{2|\lambda|}}{2}}\left(\|g\|_{L^{2}(0,1)}+\|\widetilde{g}\|_{L^{2}(0,1)}\right) .
$$

In view of $(f, \widetilde{f}, g, \widetilde{g})=\left(\lambda \operatorname{id}_{\mathscr{H}}-A\right)(\varphi, \widetilde{\varphi}, \psi, \widetilde{\psi})$, we find from (3.47), (3.48), (3.49) and (3.50) that

$$
\begin{aligned}
\|(\varphi, \widetilde{\varphi}, \psi, \widetilde{\psi})\|_{\mathscr{H}} & \leqslant C \sqrt{|\lambda|} e^{\frac{\sqrt{2|\lambda|}}{2}}\left\|\left(\lambda \operatorname{id} \operatorname{H}_{\mathscr{H}}-A\right)(\varphi, \widetilde{\varphi}, \psi, \widetilde{\psi})\right\|_{L^{2}\left(0,1 ; \mathbb{R}^{4}\right)} \\
& \leqslant C \sqrt{|\lambda|} e^{\frac{\sqrt{2|\lambda|}}{2}}\left\|\left(\lambda \operatorname{id}_{\mathscr{H}}-A\right)(\varphi, \widetilde{\varphi}, \psi, \widetilde{\psi})\right\|_{\mathscr{H}},
\end{aligned}
$$

which is equivalent to

$$
\left\|\left(\lambda \operatorname{id}_{\mathscr{H}}-A\right)^{-1}(f, \widetilde{f}, g, \widetilde{g})\right\|_{\mathscr{H}} \leqslant C \sqrt{|\lambda|} e^{\frac{\sqrt{2|\lambda|}}{2}}\|(f, \widetilde{f}, g, \widetilde{g})\|_{\mathscr{H}}
$$

Since $(f, \widetilde{f}, g, \widetilde{g})$ is arbitrary in $\mathscr{H}$, we have

$$
\left\|\left(\lambda \operatorname{id}_{\mathscr{H}}-A\right)^{-1}\right\|_{\mathscr{L}(\mathscr{H})} \leqslant C \sqrt{|\lambda|} e^{\frac{\sqrt{2|\lambda|}}{2}} \leqslant \widehat{C} e^{\widehat{C}|\lambda|}
$$

for sufficiently large constant $\widehat{C}$, say, $\widehat{C} \geqslant \max \left(\frac{\sqrt{2}}{2}, \frac{C}{2} e^{\frac{\sqrt{2}}{4}}\right)$. The proof is complete.

Lemma 3.4 (see $[11,12,13])$. Let $\check{A}$ be the infinitesimal generator of a strongly continuous semigroup $\left\{e^{t \check{A}}\right\}_{t \in[0,+\infty)}$ of contractions on a complex Hilbert space $\breve{H}$. If there there exists a $C_{1}>0$ such that

$$
\left\|\left(\lambda \operatorname{id}_{\check{H}}-\check{A}\right)^{-1}\right\|_{\mathscr{L}(\check{H})} \leqslant C_{1} e^{C_{1}|\lambda|}, \quad \forall \lambda \in \mathrm{i} \mathbb{R}
$$

then there exists a $C_{2}>0$ such that, for every $\breve{h} \in \mathscr{D}(\check{A})$,

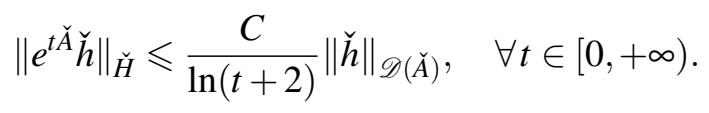

Proof of Theorem 1.2. Using Proposiiton 3.2 and Lemma 3.4, we can obtain the desired conclusion immediately. 


\section{CONCLUSION}

We investigated in this paper transmission system (1.1) of a 1-D damped wave equation and a 1-D undamped plate equation for its large time behavior. We proved that the spectrum of the infinitesimal generator of the semigroup associated to the system under consideration consists merely of eigenvalues which lie to the left of the imaginary axis. Indeed, we obtained more information on the spectrum: The spectrum has two distinct vertical lines as its asymptote lines in which the one on the right hand side is the imaginary axis. Inspired by the spectral structure, we analyze the spectrum and prove by establishing a resolvent estimate that the energy of (1.1) decreases to zero as at a logarithmic decaying rate. We conclude this paper with an interesting question: Can one obtain a polynomial decaying rate for the energy functional $E(t)$ defined by (1.4)?

\section{Acknowledgements}

The author was supported by NSFC (\#11701050 and \#11571244), and by SCJYT Program (\#18ZB0098) of Sichuan Province, China.

\section{REFERENCES}

[1] H.T. Banks, R.C. Smith, Y. Wang, Modeling aspects for piezoelectric patch activation of shells, plates and beams, Quart. Appl. Math. 53 (1995), 353-381.

[2] M.S. Alves, C.A. Raposo, J.E. Muñoz Rivera, M. Sepúlveda, O.V. Villagrán, Uniform stabilization for the transmission problem of the Timoshenko system with memory, J. Math. Anal. Appl. 369 (2010), 323-345.

[3] F. Cardoso, G. Vodev, Boundary stabilization of transmission problems, J. Math. Phys. 51 (2010), 023512.

[4] F. Hassine, Logarithmic stabilization of the Euler-Bernoulli transmission plate equation with locally distributed KelvinVoigt damping, J. Math. Anal. Appl. 455 (2017), 1765-1782.

[5] K. Ammari, S. Nicaise, Stabilization of a transmission wave/plate equation, J. Differential Equations 249 (2010), $707-727$.

[6] W. Zhang, Z.F. Zhang, Stabilization of transmission coupled wave and Euler-Bernoulli equations on Riemannian manifolds by nonlinear feedbacks, J. Math. Anal. Appl. 422 (2015), 1504-1526.

[7] F. Hassine, Asymptotic behavior of the transmission Euler-Bernoulli plate and wave equation with a localized KelvinVoigt damping, Discrete Contin. Dyn. Syst. Ser. B 21 (2016), 1757-1774.

[8] X. Fu, Q. Lu, Stabilization of the weakly coupled wave-plate system with one internal damping, http://arxiv.org/abs/1801.00232

[9] B. Gong, F. Yang, X. Zhao, Stabilization of the transmission wave/plate equation with variable coefficients, J. Math. Anal. Appl. 455 (2017), 947-962.

[10] L. Deng, Z. Zhang, Controllability for transmission wave/plate equations on Riemannian manifolds, Systems Control Lett. 91 (2016), 48-54.

[11] C.J.K. Batty, T. Duyckaerts, Non-uniform stability for bounded semi-groups on Banach spaces, J. Evol. Equ. 8 (2008), 765-780.

[12] N. Burq, Décroissance de l'énergie locale de l'équation des ondes pour le problme extérieur et absence de rsonance au voisinage du réel, Acta Math. 180 (1998), 1-29.

[13] A. Borichev, Y. Tomilov, Optimal polynomial decay of functions and operator semigroups, Math. Ann. 347 (2010), $455-$ 478.

[14] A. Pazy, Semigroups of Linear Operators and Applications to Partial Differential Equations. Springer-Verlag, Berlin (1983)

[15] B.Z. Guo, S.P. Yung, Asymptotic behavior of the eigenfrequency of a one-dimensional linear thermoelastic system, J. Math. Anal. Appl. 213(1997), 406-421. 